\title{
Design and Implementation of CUSUM Exceedance Control Charts for Unknown Location
}

\author{
MARIEN A. GRAHAM \\ Department of Statistics \\ University of Pretoria \\ South Africa \\ marien.graham@up.ac.za
}

\author{
S. CHAKRABORTI \\ Department of Information \\ Systems, Statistics and \\ Management Science, \\ University of Alabama \\ Tuscaloosa, AL 35487, \\ U.S.A. \\ schakrab@cba.ua.edu
}

\author{
AMITAVA MUKHERJEE* \\ Indian Institute of Management \\ Udaipur \\ Operations Management \\ Quantitative Techniques and \\ Information System Area \\ Rajasthan-313001, India \\ amitmukh2@yahoo.co.in
}

\begin{abstract}
Nonparametric control charts provide a robust alternative in practice when the form of the underlying distribution is unknown. Nonparametric CUSUM (NPCUSUM) charts blend the advantages of a CUSUM with that of a nonparametric chart in detecting small to moderate shifts. In this paper, we examine efficient design and implementation of Phase II NPCUSUM charts based on exceedance (EX) statistics, called the NPCUSUM-EX chart. We investigate the choice of the order statistic from the reference (Phase I) sample that defines the exceedance statistic. We see that choices other than the median, such as the $75^{\text {th }}$ percentile, can yield improved performance of the chart in certain situations. Furthermore, observing certain shortcomings of the average run-length $(A R L)$, we use the median run-length $(M R L)$ as the performance metric. The NPCUSUM-EX chart is compared with the NPCUSUM-Rank chart proposed by Li et al. (2010) based on the popular Wilcoxon rank-sum statistic. We also study the choice of the reference value, $k$, of the CUSUM charts. An illustration with real data is provided.
\end{abstract}

Keywords: Nonparametric; Robustness; Precedence; Average run-length $(A R L)$; Median run-length $(M R L)$; Order Statistics.

\section{Introduction}

Nonparametric control charts provide a robust alternative when there is lack of knowledge about the underlying distribution. A key advantage of nonparametric charts is that their in-control (IC) run-length distribution remains invariant for all continuous process distributions. This is not true for parametric control charts in general and consequently, their IC robustness can be a legitimate concern. Moreover, nonparametric charts are often more robust and efficient under some heavytailed symmetric and skewed distributions. For more details on the nonparametric control charts literature see Chakraborti and Graham (2007) and Chakraborti et al. (2011).

The Shewhart-type charts are the most widely known charts in practice because of their simplicity. Nevertheless, the cumulative sum (CUSUM) charts are useful specially for detecting small and persistent shifts. With the availability of commercial software, CUSUM charts have now moved far beyond manufacturing into biology, medicine, athletics, finance and other areas. The advantages of CUSUM charts have been well documented and the reader is referred to Khoo and Teh 
(2009) and the references therein. For a detailed discussion on CUSUM charts the reader is referred to Hawkins and Olwell (1998). More recently, Goel (2011) has summarized the basic princles and characteristics of CUSUM charts.

In this paper we study a class of Phase II nonparametric CUSUM (denoted by NPCUSUM) charts for monitoring the unknown location parameter based on the exceedance statistic which is constructed using a pre-defined order statistic from a reference sample. Although the main focus of our study is to design the most efficient NPCUSUM-EX chart using the appropriate order statistic from the Phase I reference sample, some other key questions that we address are as follows. A NPCUSUM chart using the reference sample median was first introduced and studied by Mukherjee et al. (2013). Unlike the traditional exceedance/precedence charts, instead of the reference sample median, we first investigate the efficient choice of the order statistic from an IC reference sample. More details about the exceedance/precedence statistic are given in Section 2. Second, in order to apply the NPCUSUM-EX chart, the reference value and the decision interval need to be specified. Mukherjee et al. (2013) did not provide much detail about the choice of these parameters in relation with the performance of the chart. This issue is addressed here. Finally, the performance of the NPCUSUM-EX chart is compared with a competing rank-based nonparametric chart, the NPCUSUM chart based on the Wilcoxon rank-sum statistic, proposed by Li et al. (2010) and denoted NPCUSUM-Rank chart. More details about the NPCUSUM-Rank chart are given in Appendix A.1.

Here we consider two-sided charts for detecting shifts in both directions (increasing or decreasing) unlike Mukherjee et al. (2013), who studied an upper one-sided CUSUM chart. Moreover we use the median run-length $(M R L)$, motivated by Khoo et al. (2011) and others, as the performance metric in implementing the charts. The $M R L$ has certain practical advantages over the traditional practice of using the $A R L$. More information about the benefits of $M R L$ is provided later.

The remainder of this paper is organized as follows: In Section 2, the NPCUSUM-EX chart is presented. In Section 3, the implementation of the charts is discussed and the out-of-control (OOC) chart performance of the NPCUSUM-EX charts is studied and compared to that of the NPCUSUMRank chart. An illustrative example is given in Section 4. We conclude with a summary and some recommendations.

\section{Statistical Framework and Preliminaries of CUSUM Charts}

Host of researchers have addressed nonparametric control charts for process monitoring with the specified location (Case K).Here, we consider the problem of monitoring the unknown location (Case U) of a process: (i) McDonald (1990) considered a CUSUM procedure for individual observations based on statistics called 'sequential ranks' (ii) Jones et al. (2004) discussed the runlength distribution of the CUSUM with estimated parameters and provided a method for 
approximating the distribution and moments; (iii) Chatterjee and Qiu (2009) proposed NPCUSUM charts using bootstrap control limits; (iv) Liu et al. (2013) proposed an adaptive NPCUSUM scheme for detecting unknown shifts in location and (v) Yang and Cheng (2011) proposed a NPCUSUM chart for variables data to monitor the process mean.

We consider a reference sample $X_{1}, X_{2}, \ldots, X_{m}$ from an IC process with an unknown continuous cdf $F(x)$. Let $Y_{j 1}, Y_{j 2}, \ldots, Y_{j n}, j=1,2, \ldots$, denote the $j^{\text {th }}$ test Phase II sample of size $n$ with $\operatorname{cdf} G(y)$. Both $F$ and $G$ are unknown continuous cdf's and the process is IC when $F=G$. For detecting a change in the location, we use the location model $G_{Y}(x)=F(x-\delta)$ where $\delta \epsilon(-\infty, \infty)$ is the unknown location parameter so the process is IC when $\delta=0$.

\section{NPCUSUM-EX Chart}

Let $U_{j, r}$ denote the the number of $Y$ observations in the $j^{\text {th }}$ Phase II sample that exceeds $X_{(r)}$, the $r^{\text {th }}$ ordered observation in the reference sample. The statistic $U_{j, r}$ is called an exceedance statistic and the probability $p_{r}=P\left[Y>X_{(r)} \mid X_{(r)}\right]$ is called an exceedance probability. The number of $Y$ observations in the $j^{\text {th }}$ Phase II sample that precede $X_{(r)}$ is called a precedence statistic and has been used by Chakraborti et al. (2004) to study the nonparametric Shewhart precedence chart. As in the Mukherjee et al. (2013), here we consider exceedance which may be advantageous in some cases, although for inference purposes; the exceedance and precedence tests are equivalent in the sense that the two statistics are linearly related.

Given $X_{(r)}=x_{(r)}$, it can be shown that the $U_{j, r}$ follows a binomial distribution with parameters $\left(n, p_{r}\right)$ and thus, conditionally on $X_{(r)}$, we can use a binomial-type CUSUM chart based on the $U_{j, r}$ 's to monitor the process location (via the exceedance probabilities). Noting that $E\left(U_{j, r} \mid X_{(r)}\right)=n p_{r}$ and the conditional probability $p_{r}$ is unknown, we may replace it by its unconditional IC value denoted by $d$. The latter is obtained by observing that when the process is IC, $p_{r}$ follows a beta distribution with parameters $m-r+1$ and $r$. Thus $d=E\left(p_{r} \mid \mathrm{IC}\right)=P(Y>$ $X_{(r)} \mid$ IC $)=1-\frac{r}{m+1}=\frac{m-r+1}{m+1}$. Hence the two-sided NPCUSUM-EX chart has plotting statistics

$$
C_{j}^{+}=\max \left[0, C_{j-1}^{+}+\left(U_{j, r}-n d\right)-k\right]
$$

and

$$
C_{j}^{-}=\min \left[0, C_{j-1}^{-}+\left(U_{j, r}-n d\right)+k\right]
$$

for $j=1,2,3, \ldots$, with starting values $C_{0}^{+}=C_{0}^{-}=0$ and $k \geq 0$ is the reference value. The chart signals a possible OOC situation for the first $j$ at which either $C_{j}^{-}<-H$ or $C_{j}^{+}>H$, where $H>0$ is the decision interval. Otherwise, the process is considered IC and process monitoring continues without interruption. 
The traditional NPCUSUM-EX chart is based on the median of the reference sample as the median is a robust and good representative of the reference data and one of the popular percentiles in practice. However, the question of which reference sample order statistic should be chosen to create the chart, has neverbeen examined. To this end, we investigate the performance of the NPCUSUMEX chart systematically, based on the $25^{\text {th }}, 40^{\text {th }}, 50^{\text {th }}, 60^{\text {th }}$ and $75^{\text {th }}$ percentiles, respectively. Note that for brevity, a shorthand notation is used to describe the charts. For example, the NPCUSUM-EX chart based on the $50^{\text {th }}$ percentile is denoted by $\operatorname{EX}(50)$. We also study the effect of the choice of the reference value, $k$.

\section{Implementation and Performance}

The two key quantities necessary to implement the CUSUM chart are the reference value, $k$, and the decision interval, $H$. These are typically based on the magnitude of the shift to be detected and some nominal value of an IC chart performance characteristic. The most widely used chart performance metric is the $A R L$ and determining the charting constants typically involves specifying a nominal IC $A R L$ value, such as 370 . However, since the run-length distribution is significantly rightskewed, researchers have often recommended other measures for the assessment of chart performance. More specifically, the $M R L$ provides additional and more meaningful information about the in-control and out-of-control performances of control charts compared to the $A R L$. The idea of looking at percentiles in SPC goes back to Barnard (1959). Recently Khoo et al. (2011) have advocated the use of percentiles, such as the median, for assessment of chart performance. The disadvantages of using the $A R L$ are summarized in Appendix A.2. Further, the use of the MRL will ensure a guaranteed control over the FAR in the sense that no more than $50 \%$ of the false alarms will be realized before the $M R L_{0}$. Moreover, there is practically no disadvantage of using the $M R L$ except possibly one. For the $M R L$, finding the exact standard error is not straightforward unless one uses bootstrapping or other sophisticated techniques..

When comparing the performance of charts the $M R L_{0}$ is fixed at an acceptably 'high' level and the chart with the smaller $M R L_{\delta}$ is preferred. To this end, note that the run-length distribution of a Shewhart chart follows a Geometric distribution with success probability $p=\frac{1}{A R L_{0}}$. For industry standard values for $A R L_{0}$ such as $500,=\frac{1}{500}=0.002$. Consequently, it follows that the $M R L_{0}$ equals $\frac{-1}{\log _{2}(1-0.002)}=346.2$. Therefore, keeping parity with the traditional Shewhart-type chart and for simplicity, we recommend 350 as the target nominal IC $M R L_{0}$. 


\subsection{Implementation: Chart Design Parameters}

Practical implementation of the NPCUSUM-EX charts requires specifying the following quantities: (i) $m$ : the size of the IC Phase I reference sample ${ }^{*}$, (ii) $r$ : the order of the reference sample order statistic, (iii) $n$ : the size of each Phase II test sample, (iv) $t$ : the desired $M R L_{0}$, (v) $k$ : the reference value and (vi) $H$ : the decision interval depending on $m, n, r, t$ and $k$. The experimenter need to specify the parameters $m, n, r$ and $t$ in a given situation. The choice of $r$ will be discussed later.

The design parameters $k$ and $H$ are chosen so that the chart has a specified nominal $M R L_{0}$ and is capable of detecting a specified amount of shift as soon as possible. The first step in this direction is to choose $k$. For the parametric CUSUM chart the choice of $k$ has been discussed by many authors where, typically, $k=\frac{1}{2} \delta$ with $\delta$ representing the size of the shift in the mean expressed in standard deviation units. Hawkins and Olwell (1998) investigated the sensitivity of the ARL considering four values of $k$, namely $k=0.25,0.5,1$ and 2 and concluded that the CUSUM with $k=0.25$ is the best of the four for all $\Delta<0.73$. The CUSUM with $k=0.50$ is the best in the range $0.73<\Delta<1.46$. The CUSUM with $k=1$ leads in the range $1.46<\Delta<2.87$, whereas the CUSUM with $k=2$ is the best of these four for $\Delta>2.87$. In their conclusion, $\Delta$ denotes the size of the actual mean shift ${ }^{\dagger}$. Kim et al. (2007) considered two values of the reference value $k$, namely, $k=0$ and $k=0.5$ and found that the CUSUM chart with $k=0$ is more effective in detecting shifts of size $0.25 \sigma_{Y}$, where $\sigma_{Y}$ is the process standard deviation, whereas the chart with $k=0.5$ detects any shift exceeding $0.25 \sigma_{Y}$ much faster. Recently, Mukherjee et al. (2013) examined the impact of $k$, for the parametric CUSUM chart, and concluded that when there is little or no a-priori information regarding the size of the shift a smaller value of $k$ is the safest choice to protect against any unnecessary delays in detection. They came to a similar conclusion for their proposed NPCUSUM-EX chart and recommended using a small value of $k$ or even $k=0$. Here, in order to investigate the impact of the reference value on the performance of the chart more thoroughly, we consider a number of reference values, $k_{l}=\tau_{l} \operatorname{STDEV}\left(U_{j, r}\right)$ for $l=$ $1,2,3,4$, respectively, where $\tau_{l}=c_{l} \delta, c_{l}$ is a positive constant and $\delta$ is the amount of the shift to be detected in terms of standard deviation units, $\delta=0.00(0.25) 1.00,1.50,2.00$ and 3.00. The constant $c_{l}$ ranged from small to medium to large, namely, 0.25, 0.5, 1 and 2. A similar approach is used for the NPCUSUM-Rank chart and thus, in summary, for the NPCUSUM-EX and the NPCUSUM-Rank charts we set $k_{l}$ equal to $\tau_{l} \operatorname{STDEV}\left(U_{j, r}\right)$ and $\tau_{l} \operatorname{STDEV}\left(W_{j}\right)$ for $l=1,2,3,4$, respectively. Note that

\footnotetext{
* The reference sample size affects the performance of the Phase II chart; see Appendix B of Mukherjee et al. (2013) for details.

${ }^{\dagger}$ Relationship between $\delta$ and $\Delta$ : Let $\mu_{0}$ denote the target IC mean and let $\mu_{1}$ denote the OOC value of the mean. Then $\delta$ is the size of the shift in the mean expressed in standard deviation units, i.e. $\mu_{1}=\mu_{0}+\delta \sigma$ so that $\delta=\left|\mu_{1}-\mu_{0}\right| / \sigma$. In Hawkins and Olwell (1998) $\Delta$ denotes the size of the actual mean shift, i.e. $\Delta=\delta \sigma$.
} 
although shifts as large as $\delta=3.00$ were considered in this study, the largest value of $\delta$ reported in the paper is $\delta=1.00$, since, for larger shifts, (i) the run-length characteristics of the charts tend to converge to some small values, i.e., if the shift is large enough the any of the charts will signal (almost) immediately and (ii) in Section 3.2 it is shown that for moderate to large shifts the practitioner should use the $\operatorname{EX}(75)$ chart which signals very quickly for all $k$ under consideration.

The next step is to find the decision interval $H$, in conjunction with the chosen $k$, so that a desired nominal $M R L_{0}$ is attained. Using an appropriate search algorithm (see Appendix A.3) $H$ is found so that the attained $M R L_{0}$ is less than or equal to the desired nominal $M R L_{0}$.

\subsection{Out-of-control Chart Performance Comparisons}

Our performance study includes a collection of non-normal distributions that are heavytailed, symmetric and skewed. Specifically, the distributions considered in the study are: the standard normal distribution, $N(0,1)$, the exponential distribution, $\operatorname{EXP}(1)$, which is positively skewed, the Student's $t$-distribution, $t(3)$, the Laplace (or double exponential) distribution, $D E(0,1 / \sqrt{2})$, which is standard normal like, but has heavier tails, the $\log -\operatorname{Normal}(0,1)$ distribution, denoted $\log N(0,1)$, the Symmetric Mixture Normal distribution $\left[0.6 N\left(\mu_{1}=0, \sigma_{1}=0.25\right)+0.4 N\left(\mu_{2}=0, \sigma_{2}=4\right)\right]$, denoted SymmMixN, two Asymmetric Mixture Normal distributions $\left[0.6 N\left(\mu_{1}=1, \sigma_{1}=1\right)+0.4 N\left(\mu_{2}=0\right.\right.$, $\left.\left.\sigma_{2}=4\right)\right]$ and $\left[0.6 N\left(\mu_{1}=-1, \sigma_{1}=1\right)+0.4 N\left(\mu_{2}=0, \sigma_{2}=4\right)\right]$, denoted AsymmMixN1 and AsymmMixN2, respectively. Graham et al. (2012) considered these mixture normal distributions being relatively heavier tailed than the normal and with higher kurtosis. All distributions in the study have been standardised to mean/median equals 0 and the standard deviation equals 1 so that the results are easily comparable across the distributions.

The first row of each cell in the tables shows the $M R L$ and the corresponding interquartile range $(I Q R)$ in parentheses, whereas the second row shows the values of the $5^{\text {th }}, 25^{\text {th }}, 75^{\text {th }}$ and $95^{\text {th }}$ percentiles (in this order) of the run-length distribution. For each row, i.e. for each distribution, the chart that is performing the best is indicated by use of grey shading. If any two or more blocks (in the same row) are shaded, it means that the performance of the charts is similar.

Several observations can be made from an examination of Tables 1 to 4 . The decision interval, $H$, for the NPCUSUM-EX chart is the same for the $25^{\text {th }}$ and $75^{\text {th }}$ percentiles and for the $40^{\text {th }}$ and $60^{\text {th }}$ percentiles, respectively. Note that, since the $\operatorname{STDEV}\left(U_{j, r}\right)$ is the same for the pair of percentiles $\left(M-\Delta_{1}, M+\Delta_{1}\right)$ where $M$ denotes the median and $\Delta_{1}$ is an integer between 1 and 49, the reference value $k$ would be the same (since $k$ is a function of $\operatorname{STDEV}\left(U_{j, r}\right)$ ) and, consequently, 
the decision interval $H$ would be the same (the reader is referred to Section 3.1 where the choice of the design parameters $k$ and $H$ are explained in detail).

As expected, all the run-length distribution percentiles decrease sharply with increasing shift, which is expected, indicating that NPCUSUM charts are reasonably effective in detecting shifts in location. However, the effectiveness (speed of detection) of the charts varies depending on the magnitude of the shift, the underlying process distribution and the type of the reference percentile being considered in forming the exceedance statistic.

Table 1. OOC run-length characteristics (target $M R L_{0}=350, m=100, n=5$, and $\delta=0.25$ )

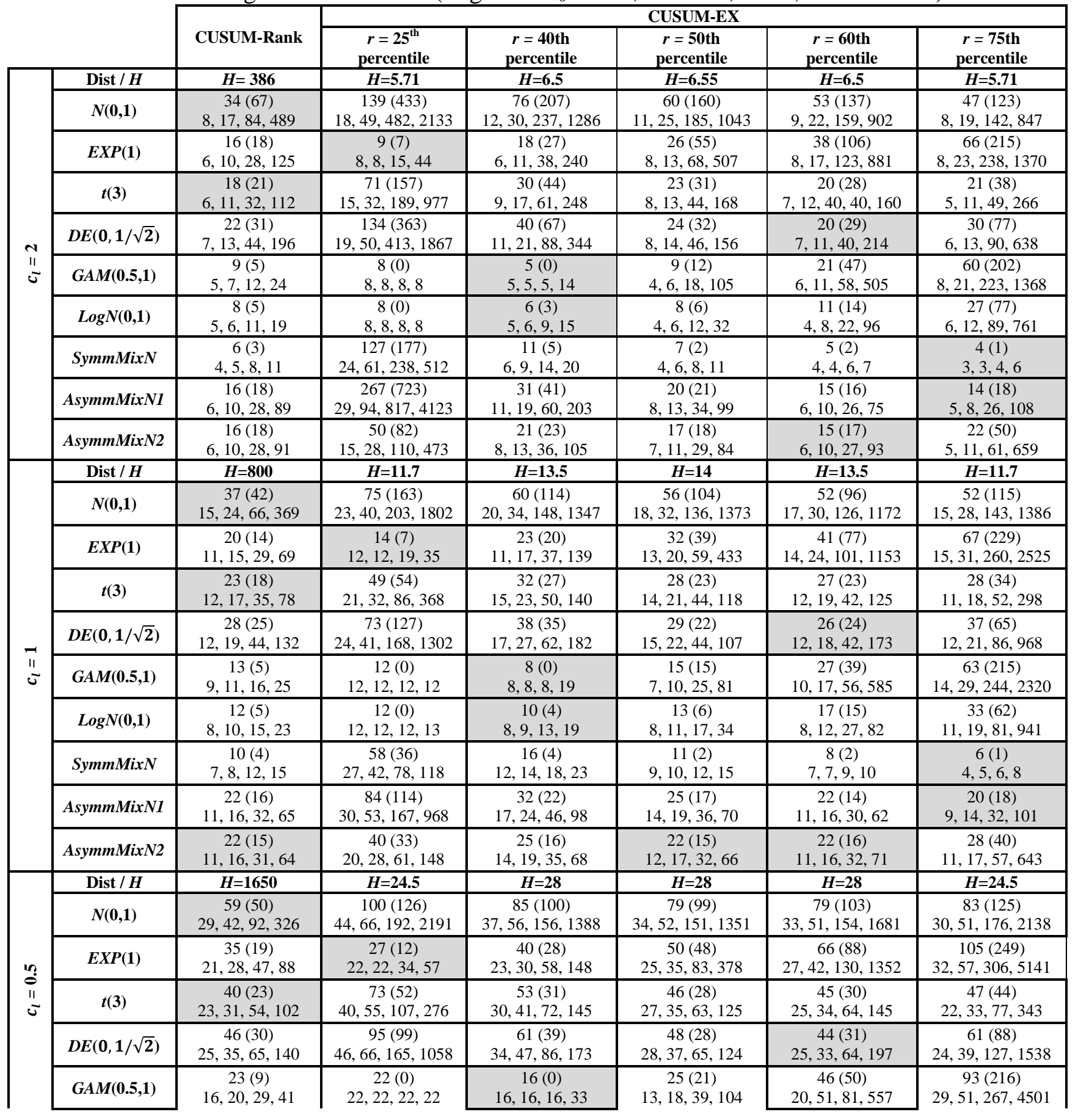




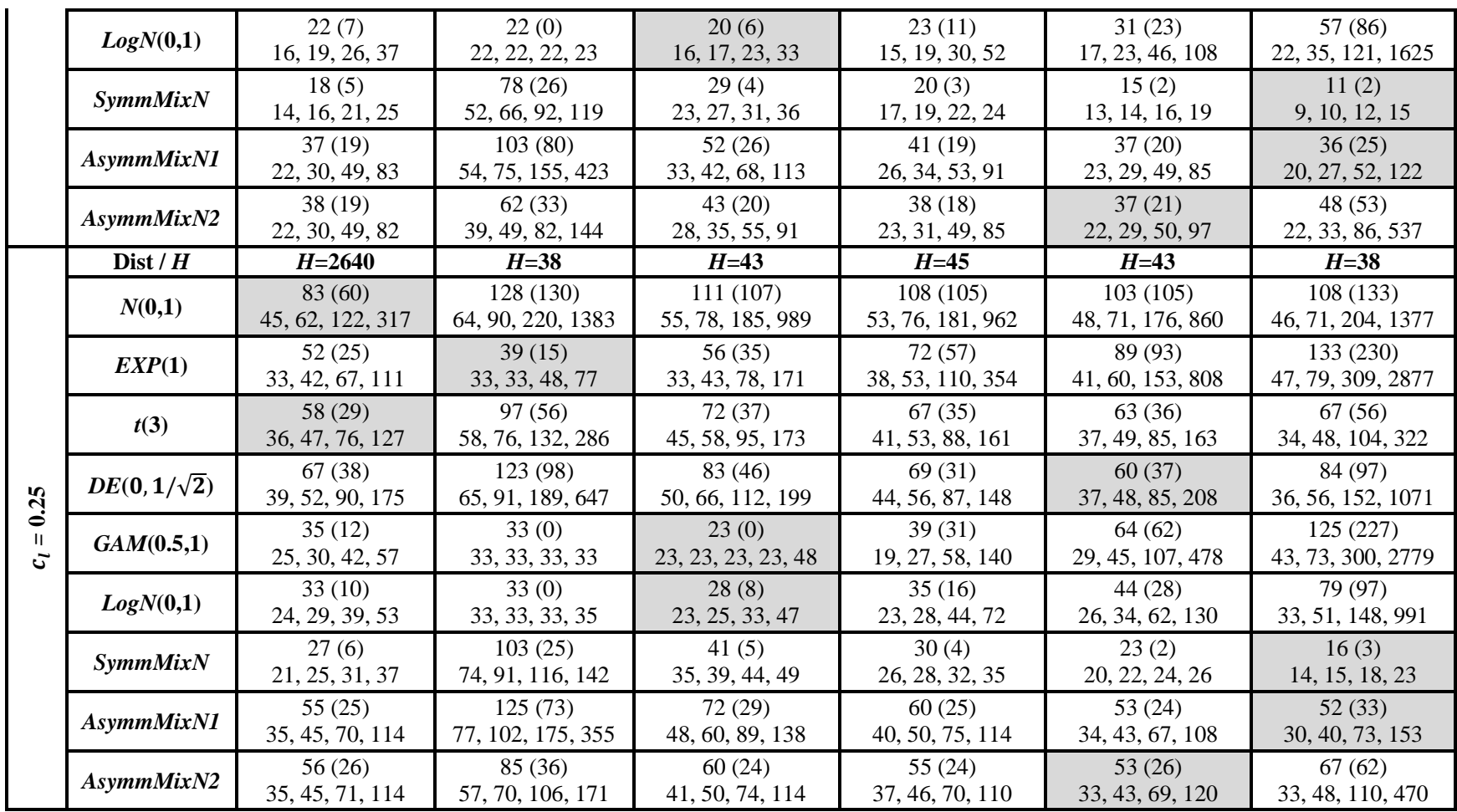

Table 2. OOC run-length characteristics (target $M R L_{0}=350, m=100, n=5$, and $\delta=0.50$ )

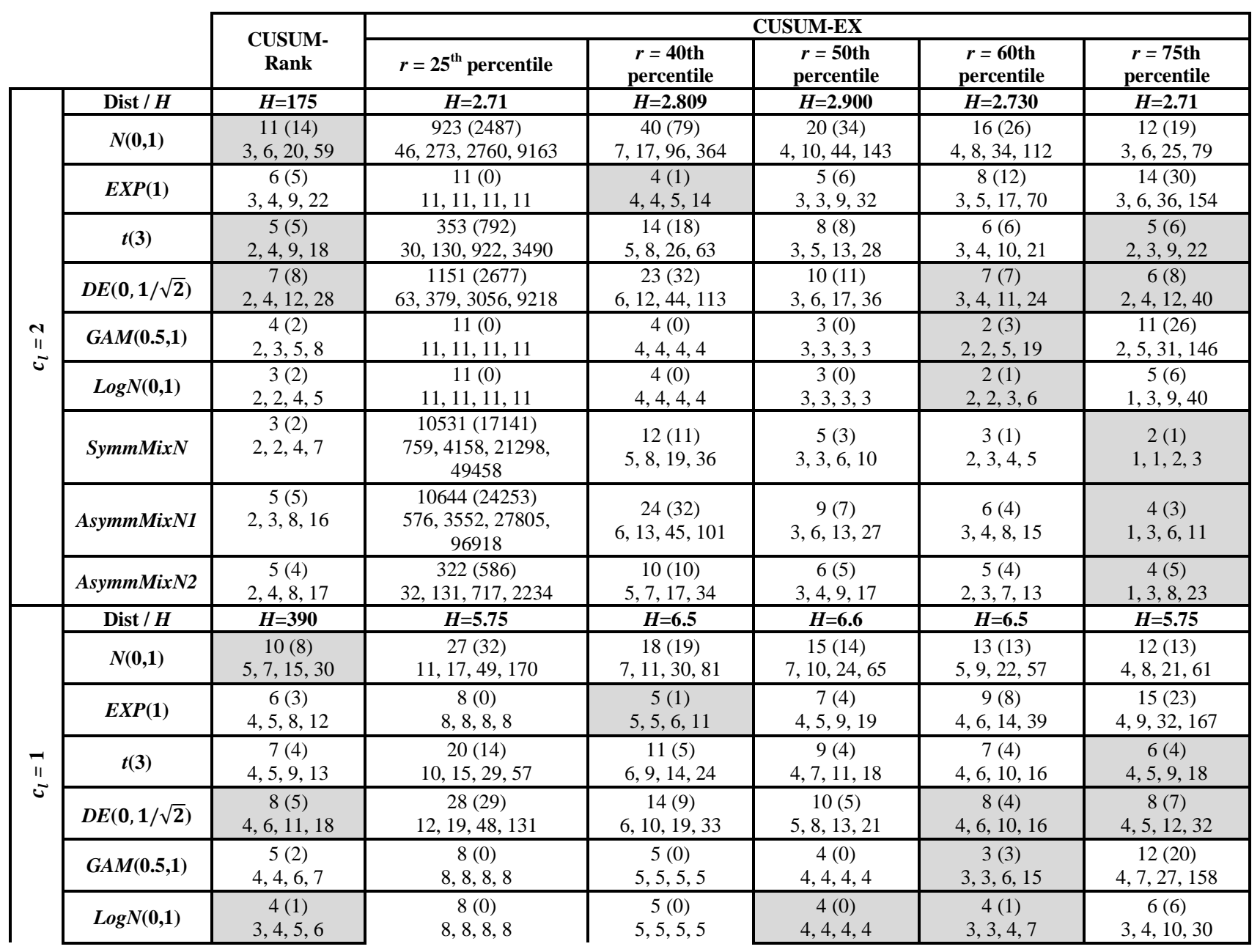




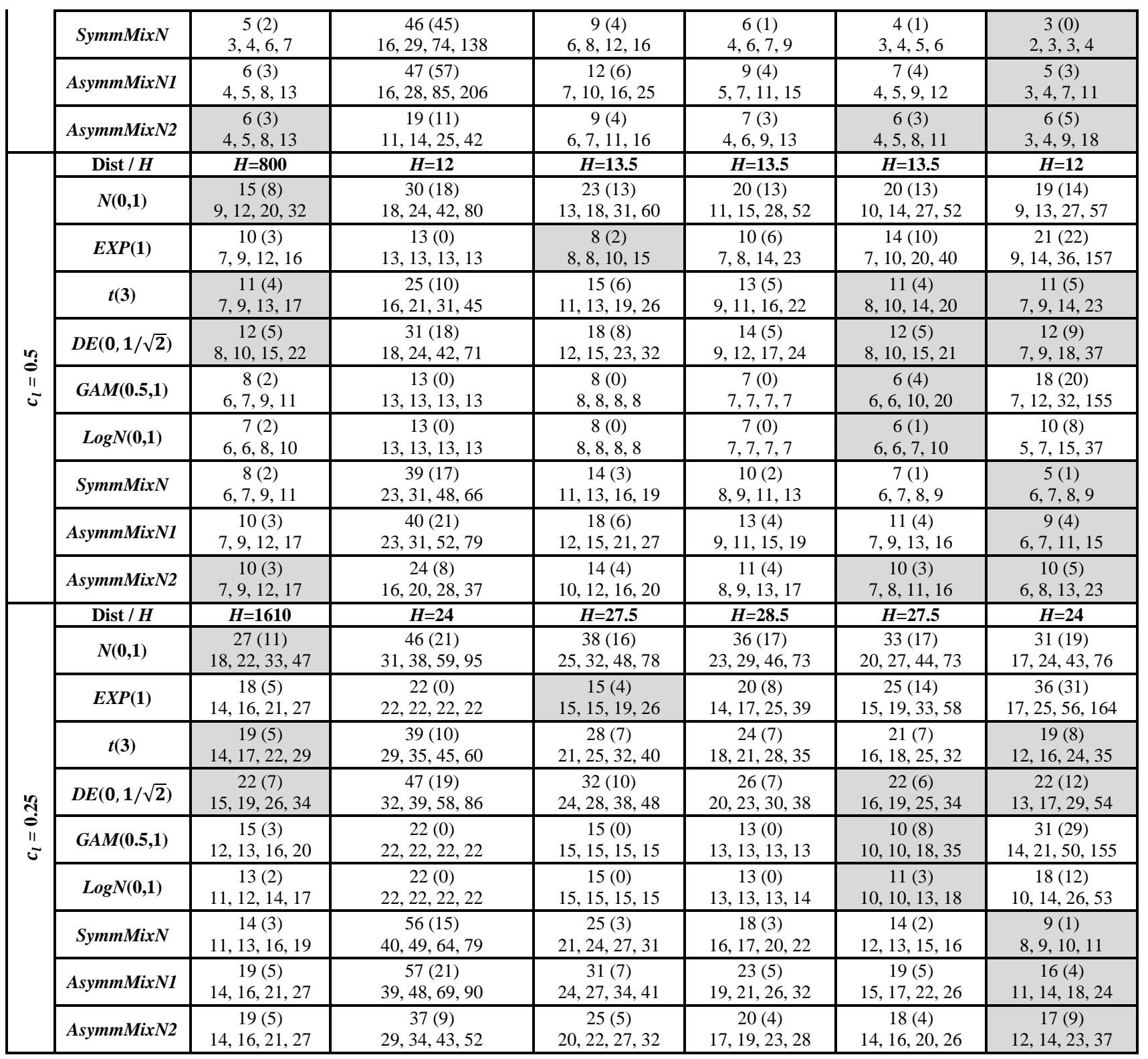

Table 3. OOC run-length characteristics (target $M R L_{0}=350, m=100, n=5$, and $\delta=0.75$ )

\begin{tabular}{|c|c|c|c|c|c|c|c|}
\hline & \multirow[b]{2}{*}{ CUSUM-Rank } & \multicolumn{5}{|c|}{ CUSUM-EX } \\
\hline & & & $\begin{array}{c}r=25^{\text {th }} \\
\text { percentile }\end{array}$ & $\begin{array}{c}r=40 \text { th } \\
\text { percentile }\end{array}$ & $\begin{array}{c}r=50 \text { th } \\
\text { percentile }\end{array}$ & $\begin{array}{c}r=60 \text { th } \\
\text { percentile }\end{array}$ & $\begin{array}{c}r=75 \text { th } \\
\text { percentile }\end{array}$ \\
\hline \multirow{9}{*}{$\begin{array}{l}\text { II } \\
\text { U }\end{array}$} & Dist / $H$ & $H=98$ & $H=1.575$ & $H=1.350$ & $H=1.530$ & $H=1.350$ & $H=1.575$ \\
\hline & $N(\mathbf{0 , 1})$ & $\begin{array}{c}6(8) \\
2,3,11,25 \\
\end{array}$ & *** & $\begin{array}{c}79(158) \\
8,31,189,675 \\
\end{array}$ & $\begin{array}{c}11(18) \\
2,5,23,59 \\
\end{array}$ & $\begin{array}{c}7(10) \\
2,4,14,33 \\
\end{array}$ & $\begin{array}{c}6(9) \\
1,3,12,31 \\
\end{array}$ \\
\hline & $E X P(1)$ & $\begin{array}{c}3(3) \\
2,2,3,13 \\
\end{array}$ & $* * *$ & $\begin{array}{c}5(0) \\
5,5,5,5 \\
\end{array}$ & $\begin{array}{c}2(0) \\
2,2,2,5 \\
\end{array}$ & $\begin{array}{c}2(2) \\
2,2,4,12 \\
\end{array}$ & $\begin{array}{c}6(10) \\
1,3,13,48 \\
\end{array}$ \\
\hline & $t(3)$ & $\begin{array}{c}3(2) \\
1,2,4,9\end{array}$ & $* * *$ & $\begin{array}{c}24(33) \\
5,12,45,105\end{array}$ & $\begin{array}{c}5(5) \\
2,3,8,17\end{array}$ & $\begin{array}{c}3(3) \\
2,2,5,9\end{array}$ & $\begin{array}{c}3(2) \\
1,2,4,8\end{array}$ \\
\hline & $D E(0,1 / \sqrt{2})$ & $\begin{array}{c}4(4) \\
1,2,6,13\end{array}$ & $* * *$ & $\begin{array}{c}48(76) \\
7,22,98,243\end{array}$ & $\begin{array}{c}7(8) \\
2,4,12,24\end{array}$ & $\begin{array}{c}4(4) \\
2,2,6,12\end{array}$ & $\begin{array}{c}3(3) \\
1,2,5,11\end{array}$ \\
\hline & $\operatorname{GAM}(0.5,1)$ & $\begin{array}{c}2(1) \\
2,2,3,5 \\
\end{array}$ & $* * *$ & $\begin{array}{c}5(0) \\
5,5,5,5 \\
\end{array}$ & $\begin{array}{c}2(0) \\
2,2,2,2 \\
\end{array}$ & $\begin{array}{c}2(0) \\
2,2,2,2 \\
\end{array}$ & $\begin{array}{c}3(6) \\
1,2,8,36 \\
\end{array}$ \\
\hline & $\log N(0,1)$ & $\begin{array}{c}2(0) \\
1,2,2,3\end{array}$ & $* * *$ & $\begin{array}{c}5(0) \\
5,5,5,5\end{array}$ & $\begin{array}{c}2(0) \\
2,2,2,2\end{array}$ & $\begin{array}{c}2(0) \\
2,2,2,2\end{array}$ & $\begin{array}{c}1(2) \\
1,1,3,6\end{array}$ \\
\hline & SymmMixN & $\begin{array}{c}2(2) \\
1,2,4,6\end{array}$ & $* * *$ & $\begin{array}{c}46(68) \\
8,22,90,186\end{array}$ & $\begin{array}{c}5(5) \\
2,3,8,15 \\
\end{array}$ & $\begin{array}{c}2(1) \\
2,2,3,5 \\
\end{array}$ & $\begin{array}{c}2(1) \\
1,1,2,3\end{array}$ \\
\hline & AsymmMixN1 & $3(3)$ & $* * *$ & $85(133)$ & $7(9)$ & $3(3)$ & $2(1)$ \\
\hline
\end{tabular}




\begin{tabular}{|c|c|c|c|c|c|c|c|}
\hline & & $1,2,5,9$ & & $10,38,171,394$ & $2,4,13,27$ & $2,2,5,10$ & $1,2,3,5$ \\
\hline & AsymmMixN2 & $\begin{array}{c}3(3) \\
1,2,5,9\end{array}$ & $* * *$ & $\begin{array}{c}19(25) \\
5,10,35,74\end{array}$ & $\begin{array}{c}4(5) \\
2,2,7,12\end{array}$ & $\begin{array}{c}2(2) \\
2,2,4,7\end{array}$ & $\begin{array}{c}2(1) \\
1,2,3,6\end{array}$ \\
\hline \multirow{10}{*}{$\begin{array}{l}\vec{I} \\
\vec{v}\end{array}$} & Dist / $H$ & $H=250$ & $H=3.5$ & $H=4.1$ & $H=4.2$ & $H=4.1$ & $H=3.5$ \\
\hline & $N(\mathbf{0 , 1})$ & $\begin{array}{c}5(3) \\
3,4,7,12\end{array}$ & $\begin{array}{c}20(20) \\
8,14,34,86\end{array}$ & $\begin{array}{c}10(7) \\
5,8,15,30\end{array}$ & $\begin{array}{c}8(5) \\
4,6,11,23\end{array}$ & $\begin{array}{c}7(6) \\
3,4,10,20\end{array}$ & $\begin{array}{c}5(4) \\
2,4,8,17\end{array}$ \\
\hline & $E X P(1)$ & $\begin{array}{c}4(1) \\
3,3,4,6\end{array}$ & $\begin{array}{c}8(0) \\
8,8,8,8\end{array}$ & $\begin{array}{c}4(0) \\
4,4,4,4\end{array}$ & $\begin{array}{c}3(0) \\
3,3,3,5\end{array}$ & $\begin{array}{c}3(3) \\
2,2,5,9\end{array}$ & $\begin{array}{c}5(6) \\
2,3,9,26\end{array}$ \\
\hline & $t(3)$ & $\begin{array}{c}3(1) \\
2,3,4,6\end{array}$ & $\begin{array}{c}16(12) \\
8,12,24,44\end{array}$ & $\begin{array}{c}7(3) \\
4,5,8,13\end{array}$ & $\begin{array}{c}5(2) \\
3,4,6,9\end{array}$ & $\begin{array}{c}4(2) \\
2,3,5,8\end{array}$ & $\begin{array}{c}3(2) \\
2,2,4,6\end{array}$ \\
\hline & $D E(0,1 / \sqrt{2})$ & $\begin{array}{c}4(2) \\
2,3,5,8 \\
\end{array}$ & $\begin{array}{c}25(26) \\
10,16,42,98 \\
\end{array}$ & $\begin{array}{c}8(4) \\
4,7,11,19 \\
\end{array}$ & $\begin{array}{c}6(3) \\
3,4,7,11 \\
\end{array}$ & $\begin{array}{c}4(3) \\
2,3,6,9 \\
\end{array}$ & $\begin{array}{c}3(2) \\
2,2,4,8 \\
\end{array}$ \\
\hline & $G A M(0.5,1)$ & $\begin{array}{c}3(0) \\
2,3,3,4 \\
\end{array}$ & $\begin{array}{c}8(0) \\
8,8,8,8 \\
\end{array}$ & $\begin{array}{c}4(0) \\
4,4,4,4 \\
\end{array}$ & $\begin{array}{c}3(0) \\
3,3,3,3\end{array}$ & $\begin{array}{c}2(0) \\
2,2,2,2 \\
\end{array}$ & $\begin{array}{c}3(4) \\
2,2,6,21 \\
\end{array}$ \\
\hline & $\log N(0,1)$ & $\begin{array}{c}3(1) \\
2,2,3,3 \\
\end{array}$ & $\begin{array}{c}8(0) \\
8,8,8,8 \\
\end{array}$ & $\begin{array}{c}4(0) \\
4,4,4,4 \\
\end{array}$ & $\begin{array}{c}3(0) \\
3,3,3,3 \\
\end{array}$ & $\begin{array}{c}2(0) \\
2,2,2,2 \\
\end{array}$ & $\begin{array}{c}2(1) \\
2,2,3,5 \\
\end{array}$ \\
\hline & SymmMixN & $\begin{array}{c}3(1) \\
2,3,4,5 \\
\end{array}$ & $\begin{array}{c}73(98) \\
16,37,135,282 \\
\end{array}$ & $\begin{array}{c}8(4) \\
4,6,10,15 \\
\end{array}$ & $\begin{array}{c}4(2) \\
3,4,6,7 \\
\end{array}$ & $\begin{array}{c}3(1) \\
2,3,4,5 \\
\end{array}$ & $\begin{array}{c}2(0) \\
2,2,2,3 \\
\end{array}$ \\
\hline & AsymmMixN1 & $\begin{array}{c}3(1) \\
2,3,4,6 \\
\end{array}$ & $\begin{array}{c}65(96) \\
14,32,128,314 \\
\end{array}$ & $\begin{array}{c}9(5) \\
5,7,12,19 \\
\end{array}$ & $\begin{array}{c}6(3) \\
4,4,7,10 \\
\end{array}$ & $\begin{array}{c}4(2) \\
2,3,5,7 \\
\end{array}$ & $\begin{array}{c}3(1) \\
2,2,3,5 \\
\end{array}$ \\
\hline & AsymmMixN2 & $\begin{array}{c}3(1) \\
2,3,4,6\end{array}$ & $\begin{array}{c}18(13) \\
8,13,26,46\end{array}$ & $\begin{array}{c}7(3) \\
4,5,8,11\end{array}$ & $\begin{array}{c}4(2) \\
3,4,6,7\end{array}$ & $\begin{array}{c}3(1) \\
2,3,4,6\end{array}$ & $\begin{array}{c}3(1) \\
2,2,3,5\end{array}$ \\
\hline \multirow{10}{*}{$\begin{array}{l}\text { n̊ } \\
0 \\
\text { II } \\
u\end{array}$} & Dist / H & $H=530$ & $H=7.8$ & $H=8.9$ & $H=8.9$ & $H=8.9$ & $H=7.8$ \\
\hline & $N(\mathbf{0 , 1})$ & $\begin{array}{c}7(3) \\
5,6,9,13 \\
\end{array}$ & $\begin{array}{c}16(7) \\
11,13,20,31 \\
\end{array}$ & $\begin{array}{c}11(6) \\
7,9,15,22 \\
\end{array}$ & $\begin{array}{c}10(5) \\
6,8,13,20 \\
\end{array}$ & $\begin{array}{c}9(5) \\
6,7,12,18 \\
\end{array}$ & $\begin{array}{c}8(5) \\
4,6,11,18 \\
\end{array}$ \\
\hline & $E X P(1)$ & $\begin{array}{c}5(1) \\
4,5,6,8 \\
\end{array}$ & $\begin{array}{c}9(0) \\
9,9,9,9 \\
\end{array}$ & $\begin{array}{c}6(0) \\
6,6,6,6 \\
\end{array}$ & $\begin{array}{c}5(0) \\
5,5,5,7 \\
\end{array}$ & $\begin{array}{c}5(3) \\
4,4,7,11 \\
\end{array}$ & $\begin{array}{c}8(6) \\
4,6,12,26 \\
\end{array}$ \\
\hline & $t(3)$ & $\begin{array}{c}5(1) \\
4,5,6,8 \\
\end{array}$ & $\begin{array}{c}14(5) \\
10,12,17,23 \\
\end{array}$ & $\begin{array}{c}9(4) \\
7,7,11,14 \\
\end{array}$ & $\begin{array}{c}7(2) \\
5,6,8,11 \\
\end{array}$ & $\begin{array}{c}6(2) \\
4,5,7,9 \\
\end{array}$ & $\begin{array}{c}5(2) \\
3,4,6,8 \\
\end{array}$ \\
\hline & $D E(0,1 / \sqrt{2})$ & $\begin{array}{c}6(2) \\
4,5,7,10 \\
\end{array}$ & $\begin{array}{c}17(8) \\
11,14,22,32 \\
\end{array}$ & $\begin{array}{c}10(4) \\
7,9,13,17 \\
\end{array}$ & $\begin{array}{c}8(2) \\
5,7,9,12 \\
\end{array}$ & $\begin{array}{c}6(2) \\
4,6,8,10 \\
\end{array}$ & $\begin{array}{c}6(3) \\
3,4,7,10 \\
\end{array}$ \\
\hline & $G A M(0.5,1)$ & $\begin{array}{c}5(1) \\
4,4,5,6 \\
\end{array}$ & $\begin{array}{c}9(0) \\
9,9,9,9 \\
\end{array}$ & $\begin{array}{c}6(0) \\
6,6,6,6 \\
\end{array}$ & $\begin{array}{c}5(0) \\
5,5,5,5 \\
\end{array}$ & $\begin{array}{c}4(0) \\
4,4,4,4 \\
\end{array}$ & $\begin{array}{c}6(5) \\
3,4,9,22 \\
\end{array}$ \\
\hline & $\log N(0,1)$ & $\begin{array}{c}4(0) \\
4,4,4,5 \\
\end{array}$ & $\begin{array}{c}9(0) \\
9,9,9,9 \\
\end{array}$ & $\begin{array}{c}6(0) \\
6,6,6,6 \\
\end{array}$ & $\begin{array}{c}5(0) \\
5,5,5,5 \\
\end{array}$ & $\begin{array}{c}4(0) \\
4,4,4,4 \\
\end{array}$ & $\begin{array}{c}3(1) \\
3,3,4,8 \\
\end{array}$ \\
\hline & SymmMixN & $\begin{array}{c}5(2) \\
4,4,6,7 \\
\end{array}$ & $\begin{array}{c}24(13) \\
14,19,32,47 \\
\end{array}$ & $\begin{array}{c}9(3) \\
7,8,11,14 \\
\end{array}$ & $\begin{array}{c}7(2) \\
5,6,8,9 \\
\end{array}$ & $\begin{array}{c}5(1) \\
4,5,6,7 \\
\end{array}$ & $\begin{array}{c}3(1) \\
3,3,4,4 \\
\end{array}$ \\
\hline & AsymmMixN1 & $\begin{array}{c}5(1) \\
4,5,6,8 \\
\end{array}$ & $\begin{array}{c}24(12) \\
14,19,31,47 \\
\end{array}$ & $\begin{array}{c}11(4) \\
7,9,13,16 \\
\end{array}$ & $\begin{array}{c}8(2) \\
6,7,9,11 \\
\end{array}$ & $\begin{array}{c}6(1) \\
4,6,7,9 \\
\end{array}$ & $\begin{array}{c}4(1) \\
3,4,5,6 \\
\end{array}$ \\
\hline & AsymmMixN2 & $\begin{array}{c}5(1) \\
4,5,6,8\end{array}$ & $\begin{array}{c}15(5) \\
11,13,18,23\end{array}$ & $\begin{array}{c}8(2) \\
6,7,9,12\end{array}$ & $\begin{array}{c}6(1) \\
5,6,7,9\end{array}$ & $\begin{array}{c}6(1) \\
4,5,6,8\end{array}$ & $\begin{array}{c}4(2) \\
3,4,6,8\end{array}$ \\
\hline \multirow{10}{*}{ 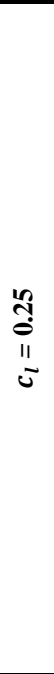 } & Dist / H & $H=1090$ & $H=16.2$ & $H=18.5$ & $H=18.5$ & $H=18.5$ & $H=16.2$ \\
\hline & $N(\mathbf{0 , 1})$ & $\begin{array}{c}13(4) \\
9,11,15,19 \\
\end{array}$ & $\begin{array}{c}24(6) \\
19,22,28,39 \\
\end{array}$ & $\begin{array}{c}19(6) \\
14,17,23,30 \\
\end{array}$ & $\begin{array}{c}17(6) \\
12,14,20,27 \\
\end{array}$ & $\begin{array}{c}16(6) \\
10,13,19,26 \\
\end{array}$ & $\begin{array}{c}14(7) \\
9,11,18,26 \\
\end{array}$ \\
\hline & $E X P(1)$ & $\begin{array}{c}10(2) \\
8,9,11,13 \\
\end{array}$ & $\begin{array}{c}33(0) \\
33,33,33,33 \\
\end{array}$ & $\begin{array}{c}11(0) \\
11,11,11,11 \\
\end{array}$ & $\begin{array}{c}9(0) \\
9,9,9,11 \\
\end{array}$ & $\begin{array}{c}10(4) \\
7,8,12,18 \\
\end{array}$ & $\begin{array}{c}14(8) \\
8,11,19,36 \\
\end{array}$ \\
\hline & $t(3)$ & $\begin{array}{c}10(2) \\
7,9,11,13 \\
\end{array}$ & $\begin{array}{c}23(4) \\
18,21,25,31 \\
\end{array}$ & $\begin{array}{c}15(3) \\
13,14,17,20 \\
\end{array}$ & $\begin{array}{c}12(3) \\
10,11,14,16 \\
\end{array}$ & $\begin{array}{c}10(2) \\
9,10,12,14 \\
\end{array}$ & $\begin{array}{c}9(2) \\
7,8,10,13 \\
\end{array}$ \\
\hline & $D E(0,1 / \sqrt{2})$ & $\begin{array}{c}11(2) \\
8,10,12,15 \\
\end{array}$ & $\begin{array}{c}26(7) \\
20,23,30,40 \\
\end{array}$ & $\begin{array}{c}17(5) \\
13,15,20,24 \\
\end{array}$ & $\begin{array}{c}14(3) \\
10,12,15,19 \\
\end{array}$ & $\begin{array}{c}12(3) \\
9,10,13,16 \\
\end{array}$ & $\begin{array}{c}10(3) \\
7,8,11,16 \\
\end{array}$ \\
\hline & $\operatorname{GAM}(0.5,1)$ & $\begin{array}{c}8(1) \\
7,8,9,10 \\
\end{array}$ & $\begin{array}{c}16(0) \\
16,16,16,16 \\
\end{array}$ & $\begin{array}{c}11(0) \\
11,11,11,11 \\
\end{array}$ & $\begin{array}{c}9(0) \\
9,9,9,9 \\
\end{array}$ & $\begin{array}{c}7(0) \\
7,7,7,7 \\
\end{array}$ & $\begin{array}{c}10(8) \\
5,7,15,21 \\
\end{array}$ \\
\hline & $\log N(0,1)$ & $\begin{array}{c}7(1) \\
6,7,8,9 \\
\end{array}$ & $\begin{array}{c}16(0) \\
16,16,16,16 \\
\end{array}$ & $\begin{array}{c}11(0) \\
11,11,11,11 \\
\end{array}$ & $\begin{array}{c}9(0) \\
9,9,9,9 \\
\end{array}$ & $\begin{array}{c}7(0) \\
7,7,7,7 \\
\end{array}$ & $\begin{array}{c}6(3) \\
5,5,8,13 \\
\end{array}$ \\
\hline & SymmMixN & $\begin{array}{c}9(2) \\
7,8,10,12 \\
\end{array}$ & $\begin{array}{c}34(11) \\
23,29,40,49 \\
\end{array}$ & $\begin{array}{c}17(3) \\
13,15,18,21 \\
\end{array}$ & $\begin{array}{c}12(2) \\
10,11,13,14 \\
\end{array}$ & $\begin{array}{c}9(1) \\
8,9,10,11 \\
\end{array}$ & $\begin{array}{c}6(1) \\
5,6,7,7 \\
\end{array}$ \\
\hline & AsymmMixN1 & $\begin{array}{c}10(2) \\
7,9,11,13 \\
\end{array}$ & $\begin{array}{c}33(12) \\
23,28,40,50 \\
\end{array}$ & $\begin{array}{c}18(4) \\
14,16,20,23 \\
\end{array}$ & $\begin{array}{c}14(2) \\
11,12,14,17 \\
\end{array}$ & $\begin{array}{c}10(2) \\
9,10,12,13 \\
\end{array}$ & $\begin{array}{c}8(2) \\
6,7,9,10 \\
\end{array}$ \\
\hline & AsymmMixN2 & $\begin{array}{c}10(2) \\
8,9,11,13 \\
\end{array}$ & $\begin{array}{c}23(4) \\
19,21,25,30 \\
\end{array}$ & $\begin{array}{c}14(2) \\
12,13,15,18 \\
\end{array}$ & $\begin{array}{c}11(2) \\
10,10,12,14 \\
\end{array}$ & $\begin{array}{c}10(1) \\
8,9,10,12 \\
\end{array}$ & $\begin{array}{c}8(2) \\
6,7,9,12 \\
\end{array}$ \\
\hline
\end{tabular}

*** Values could not be computed within a reasonable time. This indicates that the values are extremely large and the corresponding chart is performing poorly. 
Table 4. OOC run-length characteristics (target $M R L_{0}=350, m=100, n=5$, and $\delta=1.00$ )

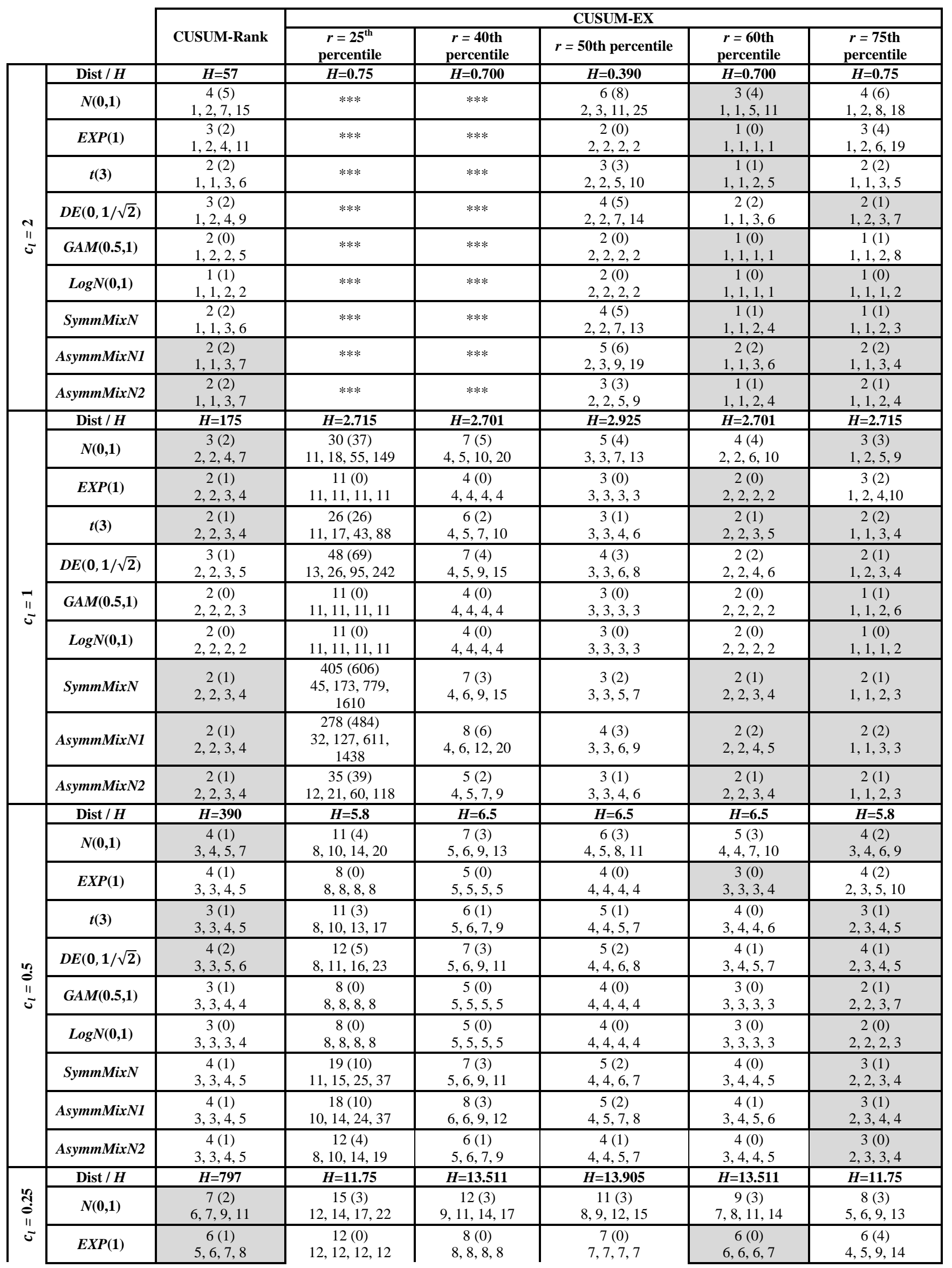




\begin{tabular}{|c|c|c|c|c|c|c|}
\hline$t(3)$ & $\begin{array}{c}6(2) \\
5,5,7,8 \\
\end{array}$ & $\begin{array}{c}15(3) \\
12,14,17,20\end{array}$ & $\begin{array}{c}10(1) \\
8,10,11,13\end{array}$ & $\begin{array}{c}8(1) \\
7,8,9,11\end{array}$ & $\begin{array}{c}7(2) \\
6,6,8,9 \\
\end{array}$ & $\begin{array}{c}5(1) \\
4,5,6,7\end{array}$ \\
\hline$D E(0,1 / \sqrt{2})$ & $\begin{array}{c}7(1) \\
5,6,7,9\end{array}$ & $\begin{array}{c}17(4) \\
13,15,19,24\end{array}$ & $\begin{array}{c}11(3) \\
9,10,13,15\end{array}$ & $\begin{array}{c}9(3) \\
7,8,11,12\end{array}$ & $\begin{array}{c}7(1) \\
6,7,8,10\end{array}$ & $\begin{array}{c}6(1) \\
4,5,6,8\end{array}$ \\
\hline $\operatorname{GAM}(0.5,1)$ & $\begin{array}{c}6(1) \\
5,5,6,7\end{array}$ & $\begin{array}{c}12(0) \\
12,12,12,12\end{array}$ & $\begin{array}{c}8(0) \\
8,8,8,8\end{array}$ & $\begin{array}{c}7(0) \\
7,7,7,7\end{array}$ & $\begin{array}{c}6(0) \\
6,6,6,6\end{array}$ & $\begin{array}{c}4(1) \\
4,4,5,11\end{array}$ \\
\hline $\log N(0,1)$ & $\begin{array}{c}5(0) \\
5,5,5,6 \\
\end{array}$ & $\begin{array}{c}12(0) \\
12,12,12,12 \\
\end{array}$ & $\begin{array}{c}8(0) \\
8,8,8,8 \\
\end{array}$ & $\begin{array}{c}7(0) \\
7,7,7,7 \\
\end{array}$ & $\begin{array}{c}6(0) \\
6,6,6,6 \\
\end{array}$ & $\begin{array}{c}4(0) \\
4,4,4,4 \\
\end{array}$ \\
\hline SymmMixN & $\begin{array}{c}6(2) \\
5,5,7,8\end{array}$ & $\begin{array}{c}22(7) \\
15,19,26,34\end{array}$ & $\begin{array}{c}12(2) \\
10,11,13,15\end{array}$ & $\begin{array}{c}9(2) \\
7,8,10,11\end{array}$ & $\begin{array}{c}7(1) \\
6,6,7,8\end{array}$ & $\begin{array}{c}4(1) \\
4,4,5,5\end{array}$ \\
\hline AsymmMixN1 & $\begin{array}{c}6(1) \\
5,6,7,8\end{array}$ & $\begin{array}{c}21(7) \\
15,18,25,33\end{array}$ & $\begin{array}{c}13(3) \\
10,11,14,16\end{array}$ & $\begin{array}{c}10(2) \\
8,9,11,12\end{array}$ & $\begin{array}{c}7(1) \\
6,7,8,9\end{array}$ & $\begin{array}{c}5(1) \\
4,5,6,6\end{array}$ \\
\hline AsymmMixN2 & $\begin{array}{c}6(1) \\
5,6,7,8\end{array}$ & $\begin{array}{c}16(4) \\
13,14,18,21\end{array}$ & $\begin{array}{c}10(1) \\
8,10,11,13\end{array}$ & $\begin{array}{c}8(1) \\
7,8,9,10\end{array}$ & $\begin{array}{c}7(1) \\
6,6,7,8\end{array}$ & $\begin{array}{c}5(1) \\
4,4,5,6\end{array}$ \\
\hline
\end{tabular}

*** Values could not be computed within a reasonable time. This indicates that the values are extremely large and the corresponding chart is performing poorly.

The observations from Tables 1 to 4 are summarized in Table 5 along with some recommendations. Note that if two charts perform similarly, for example, if the NPCUSUM-EX chart based on the $50^{\text {th }}$ and $60^{\text {th }}$ percentiles perform similarly, the notation $\operatorname{EX}(50,60)$ is used.

Table 5. Summary of the efficacy of different reference sample percentiles for the NPCUSUM-EX chart

\begin{tabular}{|c|c|c|c|c|}
\hline & \multicolumn{4}{|c|}{ Size of Location Shift } \\
\hline. $\overrightarrow{\tilde{a}}$ & $\begin{array}{c}\text { Small } \\
(\delta \leq 0.25)\end{array}$ & $\begin{array}{l}\text { Small to moderate } \\
(0.25<\delta \leq 0.50)\end{array}$ & $\begin{array}{l}\text { Moderate to large } \\
(0.50<\delta \leq 0.75)\end{array}$ & $\begin{array}{c}\text { Large } \\
(0.75<\delta \leq 1.00)\end{array}$ \\
\hline \multicolumn{5}{|c|}{ Symmetric distributions } \\
\hline$\stackrel{\vec{e}}{e_{z}^{\prime}}$ & $\begin{array}{l}\text { The Rank chart } \\
\text { performs the best for } \\
\text { all } c_{l}\end{array}$ & $\begin{array}{l}\text { The Rank chart performs } \\
\text { the best for all } c_{l}\end{array}$ & $\begin{array}{l}\text { The Rank chart } \\
\text { performs the best for } c_{l} \\
=0.25 \text { and } 0.5 . \text { For } \\
\text { larger values of } c_{l}=1 \\
\text { and } 2 \text { the Rank and } \\
\text { EX(75) charts perform } \\
\text { similarly }\end{array}$ & $\begin{array}{l}\text { The Rank chart and the } \\
\text { higher order percentile } \\
\text { based charts, EX }(75) \text { and } \\
\text { EX }(60) \text {, perform best. } \\
\text { More specifically, for each } \\
c_{l} \text {, the chart performing the } \\
\text { best is: } \\
c_{l}=2: \operatorname{EX}(60) \\
c_{l}=1: \text { Rank, EX(75) } \\
c_{l}=0.5: \text { Rank, EX }(75) \\
c_{l}=0.25: \text { Rank }\end{array}$ \\
\hline$\widehat{\overbrace{}}$ & $\begin{array}{l}\text { The Rank chart } \\
\text { performs the best for } \\
\text { all } c_{l}\end{array}$ & $\begin{array}{l}\text { The Rank and the } \\
\text { EX }(60,75) \text { charts perform } \\
\text { almost similarly and the } \\
\text { best. More specifically, for } \\
\text { each } c_{l} \text {, the chart } \\
\text { performing the best is: } \\
c_{l}=2: \text { Rank, } \operatorname{EX}(75) \\
c_{l}=1: \operatorname{EX}(75) \\
c_{l}=0.5: \text { Rank, EX }(60,75) \\
c_{l}=0.25: \text { Rank, } \operatorname{EX}(75)\end{array}$ & $\begin{array}{l}\text { The Rank and the } \\
\text { EX(75) charts perform } \\
\text { similarly for all } c_{l} \\
\text { except for } c_{l}=0.25 \\
\text { where the EX(75) chart } \\
\text { performs best. }\end{array}$ & $\begin{array}{l}\text { The Rank chart and the } \\
\text { higher order percentile } \\
\text { based charts, EX }(75) \text { and } \\
\text { EX }(60) \text {, perform best. } \\
\text { More specifically, for each } \\
c_{l} \text {, the chart performing the } \\
\text { best is: } \\
c_{l}=2: \operatorname{EX}(60) \\
c_{l}=1: \operatorname{Rank}, \operatorname{EX}(60,75) \\
c_{l}=0.5: \operatorname{Rank}, \operatorname{EX}(75) \\
c_{l}=0.25: \operatorname{EX}(75)\end{array}$ \\
\hline
\end{tabular}




\begin{tabular}{|c|c|c|c|c|}
\hline 命 & $\begin{array}{l}\text { The EX(60) chart } \\
\text { performs the best for } \\
\text { all } c_{l}\end{array}$ & $\begin{array}{l}\text { The Rank and the } \\
\text { EX }(60,75) \text { charts perform } \\
\text { similarly and the best for } \\
\text { all } c_{l}\end{array}$ & $\begin{array}{l}\text { The EX(75) chart } \\
\text { performs the best for } \\
\text { most } c_{l} \text {, however, for } \\
c_{l}=0.5 \text {, the Rank and } \\
\text { the EX }(60,75) \text { charts } \\
\text { perform similarly and } \\
\text { the best }\end{array}$ & $\begin{array}{l}\text { The Rank chart and the } \\
\text { higher order percentile } \\
\text { based charts, EX(75) and } \\
\text { EX(60), perform best. } \\
\text { More specifically, for each } \\
c_{l} \text {, the chart performing the } \\
\text { best is: } \\
c_{l}=2: \operatorname{EX}(75) \\
c_{l}=1: \operatorname{EX}(75) \\
c_{l}=0.5: \operatorname{Rank}, \operatorname{EX}(60,75) \\
c_{l}=0.25: \operatorname{EX}(75)\end{array}$ \\
\hline 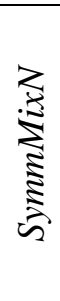 & $\begin{array}{l}\text { The EX(75) chart } \\
\text { performs the best for } \\
\text { all } c_{l}\end{array}$ & $\begin{array}{l}\text { The EX(75) chart performs } \\
\text { the best for all } c_{l}\end{array}$ & $\begin{array}{l}\text { The EX(75) chart } \\
\text { performs the best for } \\
\text { all } c_{l}\end{array}$ & $\begin{array}{l}\text { The EX(75) chart performs } \\
\text { the best for all } c_{l} \text {, except } \\
\text { for } c_{l}=1 \text { where the Rank } \\
\text { and EX }(60,75) \text { charts } \\
\text { perform similarly and } c_{l}= \\
2 \text { where the EX }(60,75) \\
\text { charts perform similarly }\end{array}$ \\
\hline \multicolumn{5}{|c|}{ Asymmetric distributions } \\
\hline$\underset{\Xi}{\nexists}$ & $\begin{array}{l}\text { The } \mathrm{EX}(25) \text { chart } \\
\text { performs the best for all } \\
c_{l}\end{array}$ & $\begin{array}{l}\text { The EX(40) chart } \\
\text { performs the best for all } \\
c_{l}\end{array}$ & $\begin{array}{l}\text { The EX }(50) \text { chart has } \\
\text { the overall advantage, } \\
\text { though the EX }(60) \\
\text { chart is equally good } \\
\text { for higher values of } c_{l} \text {. } \\
\text { The Rank chart can } \\
\text { also be used for } c_{l}= \\
0.5 \text {. }\end{array}$ & $\begin{array}{l}\text { The Rank chart and the } \\
\text { higher order percentile } \\
\text { based charts, EX(75) and } \\
\text { EX(60), perform best. } \\
\text { More specifically, for each } \\
c_{l} \text {, the chart performing the } \\
\text { best is: } \\
c_{l}=2: \operatorname{EX}(60) \\
c_{l}=1: \operatorname{Rank}, \operatorname{EX}(60) \\
c_{l}=0.5: \operatorname{EX}(60) \\
c_{l}=0.25: \operatorname{Rank}, \operatorname{EX}(60)\end{array}$ \\
\hline 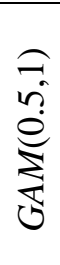 & $\begin{array}{l}\text { The EX(40) chart } \\
\text { performs the best for all } \\
c_{l}\end{array}$ & $\begin{array}{l}\text { The EX }(60) \text { chart } \\
\text { performs the best for all } \\
c_{l}\end{array}$ & $\begin{array}{l}\text { The EX(60) chart } \\
\text { performs the best for } \\
\text { all } c_{l} \text {, except for } c_{l}=2 \\
\text { where EX }(50) \text { and } \\
\text { EX(60) perform } \\
\text { similarly }\end{array}$ & $\begin{array}{l}\text { The EX(75) chart performs } \\
\text { the best for all } c_{l} \text {, except } \\
\text { for } c_{l}=2 \text { where } \operatorname{EX}(60) \\
\text { performs best }\end{array}$ \\
\hline 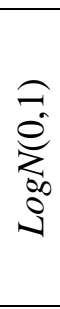 & $\begin{array}{l}\text { The EX(40) chart } \\
\text { performs the best for all } \\
c_{l}\end{array}$ & $\begin{array}{l}\text { The EX(60) chart } \\
\text { performs the best for } \\
\text { most } c_{l} \text {, however, for } \\
c_{l}=1 \text {, the Rank and the } \\
\text { EX }(50,60) \text { charts } \\
\text { perform similarly and } \\
\text { the best }\end{array}$ & $\begin{array}{l}\text { The } \mathrm{EX}(75) \text { chart } \\
\text { performs the best for } \\
\text { most } c_{l} \text {, however, for } \\
c_{l}=1 \text {, the } \mathrm{EX}(60,75) \\
\text { charts perform } \\
\text { similarly and the best }\end{array}$ & $\begin{array}{l}\text { The EX(75) chart performs } \\
\text { the best for all } c_{l} \text {, except } \\
\text { for } c_{l}=2 \text { where the } \\
\text { EX }(75) \text { and EX }(60) \text { charts } \\
\text { perform similarly }\end{array}$ \\
\hline 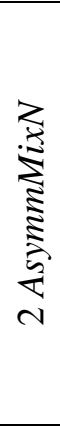 & $\begin{array}{l}\text { The EX }(75) \text { chart } \\
\text { performs the best for } \\
\text { AsymmMixN1, whereas } \\
\text { the EX(60) chart is } \\
\text { preferable for } \\
\text { AsymmMixN2. Note that } \\
\text { the Rank and EX(50) } \\
\text { charts can also be used } \\
\text { for AsymmMixN2 when } \\
c_{l}=1 \text {. }\end{array}$ & $\begin{array}{l}\text { The Rank chart and the } \\
\text { EX }(60,75) \text { charts } \\
\text { perform similarly and } \\
\text { the best for } \\
\text { AsymmMixN2 for all } c_{l} \\
\text { except for } c_{l}=0.25 \\
\text { where EX(75) performs } \\
\text { best. For AsymmMixN1 } \\
\text { the EX(75) chart always } \\
\text { performs best for all } c_{l} \text {. }\end{array}$ & $\begin{array}{l}\text { The EX(75) chart } \\
\text { performs the best for } \\
\text { all } c_{l}=0.25 \text { and } 0.5 \text {. } \\
\text { For larger values of } c_{l} \\
=1 \text { and } 2 \text { then Rank } \\
\text { and EX(60) charts can } \\
\text { also be implemented. }\end{array}$ & $\begin{array}{l}\text { The EX(75) chart performs } \\
\text { the best for all } c_{l} \text {, except } \\
\text { for } c_{l}=1 \text { and } 2 \text { where the } \\
\text { Rank and EX }(60,75) \text { charts } \\
\text { perform similarly }\end{array}$ \\
\hline
\end{tabular}


From Table 5, we find that a NPCUSUM-EX chart using the reference sample percentiles other than the median can provide a useful nonparametric chart in practice. In fact, it appears that the EX(75) chart is a good overall chart for detecting a larger location shift for a wide class of distributions. This finding is an important contribution to the literature on exceedance/precedence tests and charts. Other reference sample percentiles, such as the $25^{\text {th }}$ or the $40^{\text {th }}$, can also be used when a small shift is expected. It is worth mentioning that we recommend avoiding the choice of percentiles lower than the $25^{\text {th }}$ or higher than the $75^{\text {th }}$ percentile as there may be some problems with extreme values and higher variances, particularly for heavy-tailed distributions. Therefore, our recommendation is that the NPCUSUM-EX chart should be constructed choosing the appropriate order statistic from the Phase I sample that lies within the $25^{\text {th }}$ and $75^{\text {th }}$ percentile, both inclusive. Moreover, when we extend the comparison of the NPCUSUM-EX chart with the NPCUSUM-Rank chart, we find that the former performs better than the latter for some choices of the reference sample percentiles, for various well-known process distributions.

\section{Choice of Reference Value}

The reference value $k$ is an important parameter of a CUSUM chart and is generally chosen based on the amount of the expected shift. In the parametric context, given the population distribution, one can choose the optimal $k$ from the notion of a sequential probability ratio test; see Hawkins and Olwell (1998). Traditionally, for the parametric CUSUM chart it is recommended that smaller (larger) values of the reference value are preferable for detecting smaller (larger) shifts. In the nonparametric setting, however, the choice of such $k$ is more complicated and we investigate this issue more thoroughly, starting with revisiting the parametric CUSUM chart. Note that when there is an expected shift, the goal is to choose that value of $k$ which gives the shortest $M R L_{\delta}$. Our simulation study suggests that for both parametric and nonparametric charts, when small shifts are under consideration, the $M R L_{\delta}$ values initially decrease, as the reference value $k$ increases from 0 , reaches a minimum and then sharply increases. Thus, roughly, the $M R L_{\delta}$ is a U-shaped function as a function of $k$. Since the $M R L_{0}$ is fixed at the same value, the $k$ (or equivalently the $c_{l}$ value) with the smallest or lowest $M R L_{\delta}$ is selected to be the winner. For the parametric CUSUM chart the U-shape (with $A R L_{\delta}$ on the vertical axis against $k$ on the horizontal axis) is illustrated graphically in Figure 1 of Mukherjee et al. (2013). For the NPCUSUM-EX chart we illustrate the shape of the $M R L_{\delta}$ profile graphically in Figures 1 to 8 for different selected percentiles of the Phase I reference sample. In order to make the graphs more visually appealing, we separate the symmetric (see Figures 1 to 5) and asymmetric (see Figures 6 to 8 ) distributions. To summarize, in the nonparametric setting, the choice of $k$ is not straightforward for an exceedance chart, since the choice depends on the reference sample 
Figure 1. Out-of-control $M R L_{\delta}$ values for different symmetric distributions and under different choices of $c_{l}$ for the NPCUSUM-EX chart based on the $25^{\text {th }}$ percentile of the reference sample

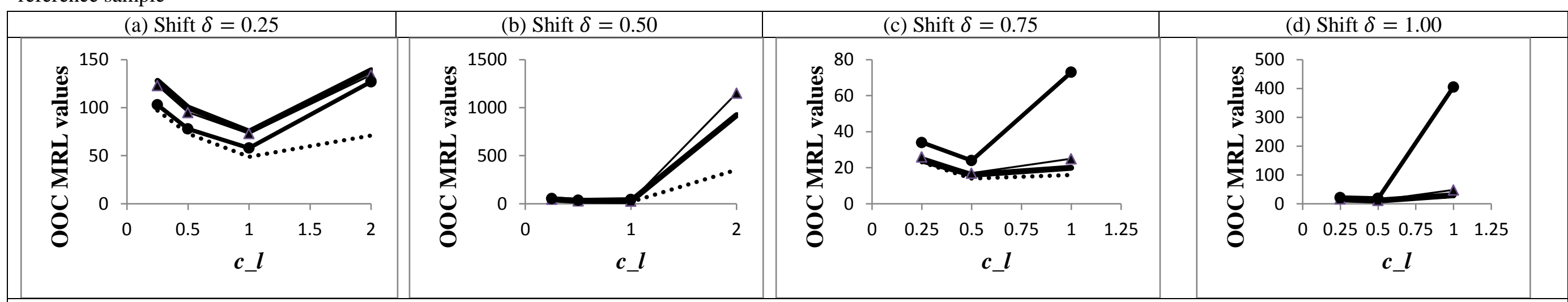

\section{Notes on Figures:}

Figure $1 \mathrm{~b}$. There is no marker for the SymmMixN distribution at $c_{l}=2$, since the run-length characteristics could not be computed within a reasonable time, indicating that the run-length characteristics are extremely large and, consequently, $c_{l}=2$ would not be chosen

Figures $1 \mathrm{c}$ and $\mathrm{d}$. There are no markers for $c_{l}=2$, at any of the distributions. Reasoning follows along the lines as the note above.

Legend. $\longrightarrow \mathrm{N}(0,1) \cdots \cdots+\mathrm{t}(3) \longrightarrow$ Double Exp $\longrightarrow$ SymmM ixN

Figure 2. Out-of-control $M R L_{\delta}$ values for different symmetric distributions under different choices of $c_{l}$ for the NPCUSUM-EX chart based on the $40^{\text {th }}$ percentile of the reference sample

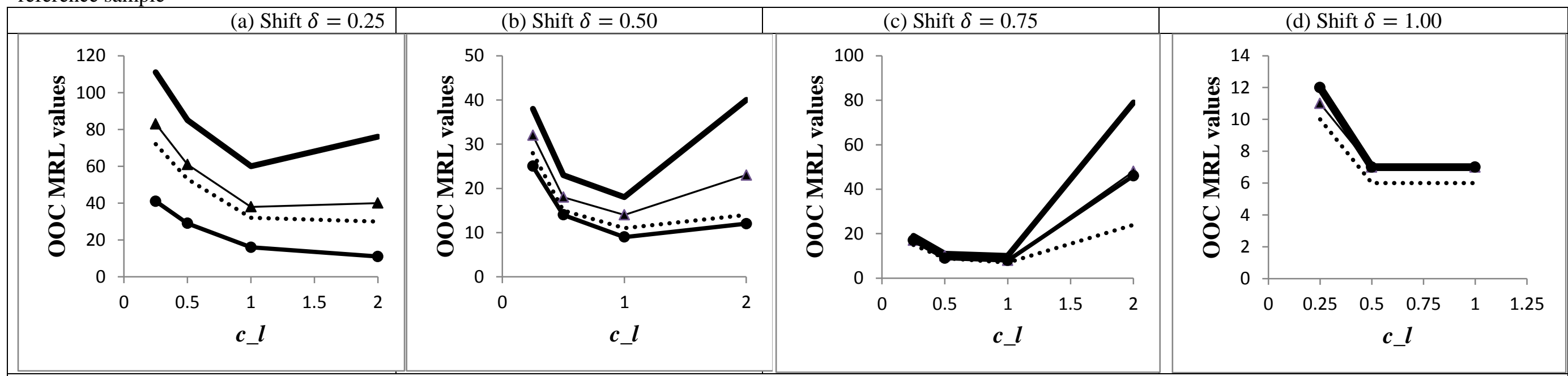

Note on Figure 2d. There is no marker for $c_{l}=2$, since the run-length characteristics could not be computed within a reasonable time, indicating that the run-length characteristics are extremely large and, consequently, $c_{l}=2$ would not be chosen.

Legend. $\mathrm{N}(0,1) \cdots \cdots+\mathrm{t}(3) \longrightarrow$ Double Exp $\longrightarrow$ SymmM ixN 
Figure 3. Out-of-control $M R L_{\delta}$ values for different symmetric distributions under different choices of $c_{l}$ for the NPCUSUM-EX chart based median of the reference sample

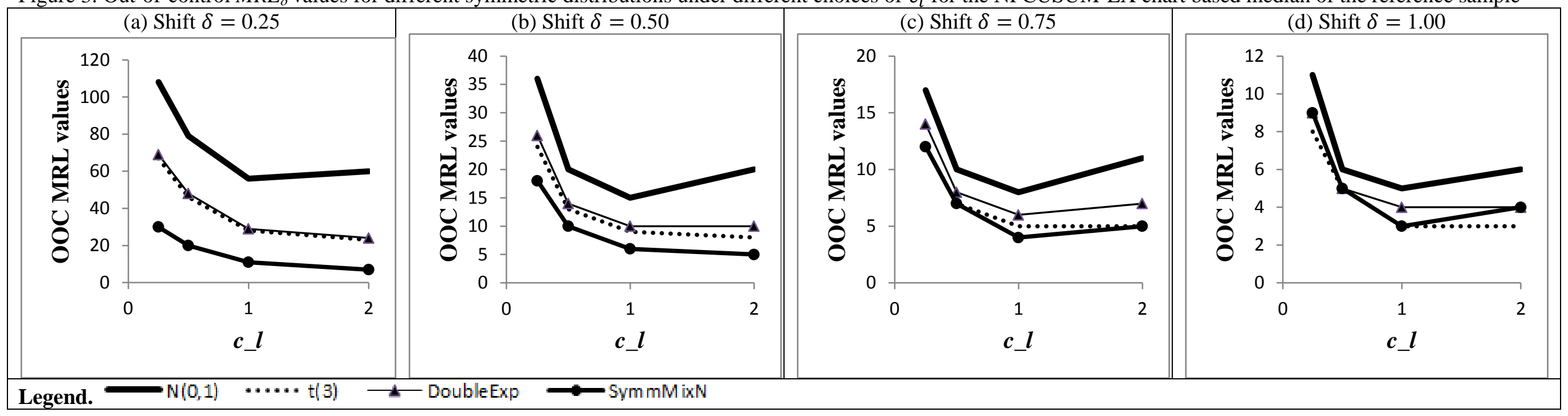

Figure 4. Out-of-control $M R L_{\delta}$ values for different symmetric distributions under different choices of $c_{l}$ for the NPCUSUM-EX chart based on the $60^{\text {th }}$ percentile of the reference sample

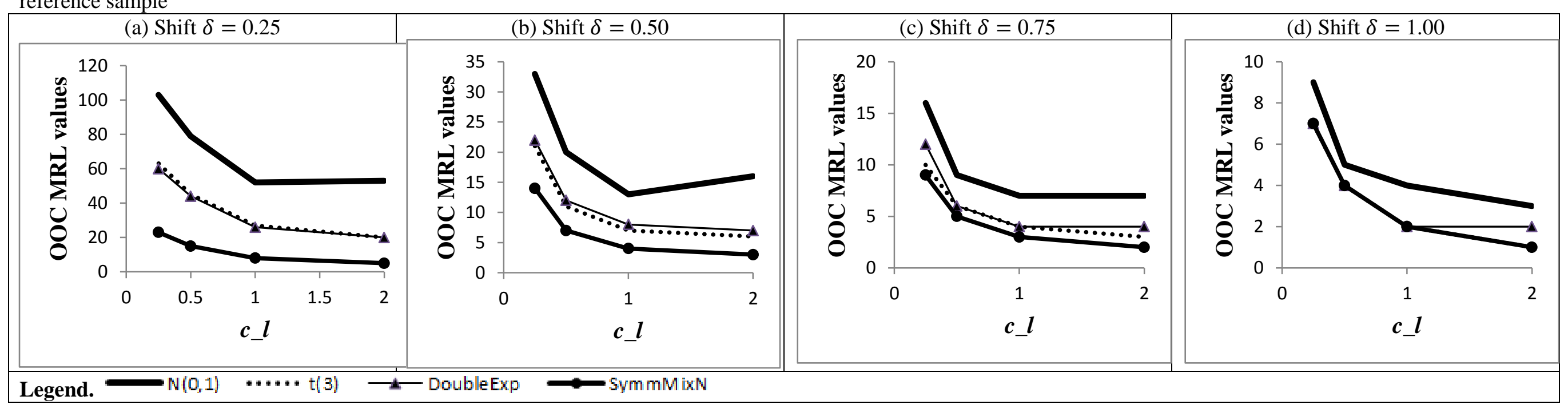


Figure 5. Out-of-control $M R L_{\delta}$ values for different symmetric distributions under different choices of $c_{l}$ for the NPCUSUM-EX chart based on the $75^{\text {th }}$ percentile of the reference sample

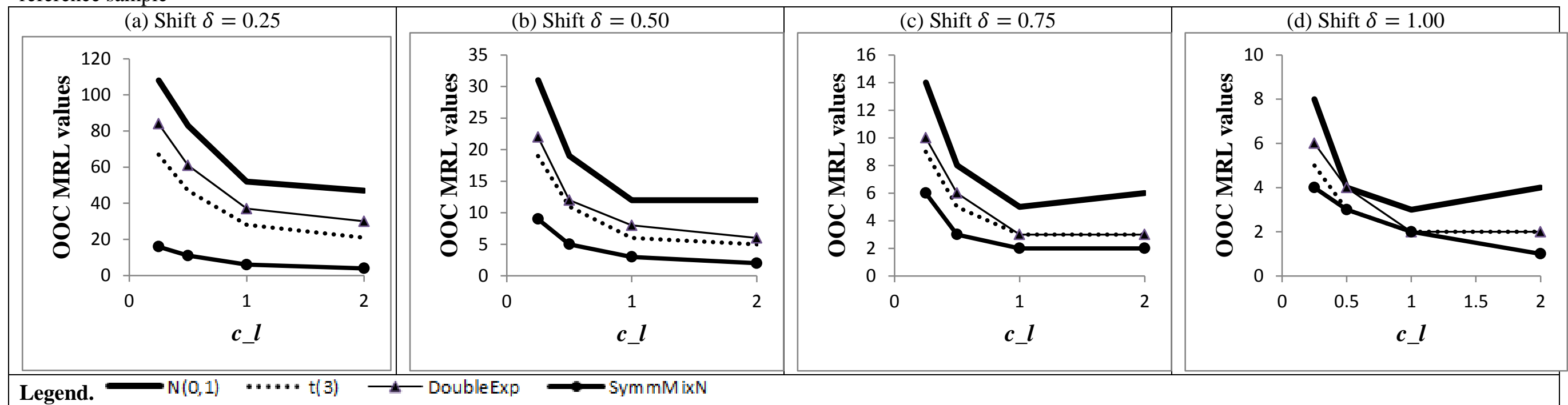

Figure 6. Out-of-control $M R L_{\delta}$ values for different asymmetric distributions and under different choices of $c_{l}$ for the NPCUSUM-EX chart based on the $25^{\text {th }}$ percentile of the reference sample

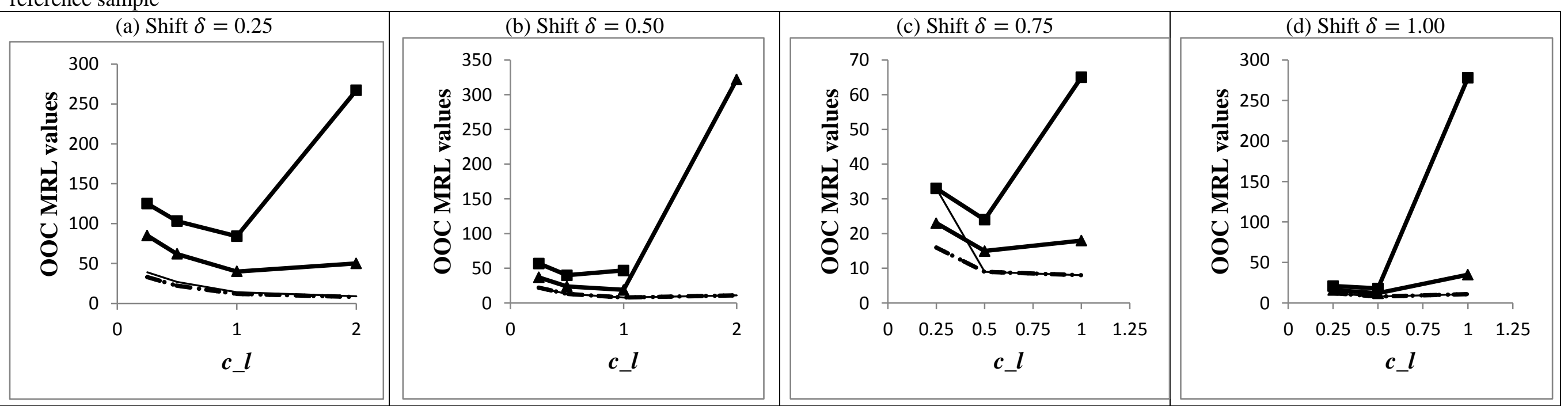

\section{Notes on Figures:}

Figure $6 \mathrm{~b}$. The is no marker for the AsymmMixN1 distribution at $c_{l}=2$, since the run-length characteristics could not be computed within a reasonable time, indicating that the run-length characteristics are extremely large and, consequently, $c_{l}=2$ would not be chosen

Figures $6 \mathrm{c}$ and $\mathrm{d}$. There are no markers for $c_{l}=2$, at any of the distributions. Reasoning following the same lines as the note above.

Legend. 
order statistic, the magnitude of shift that one wants to detect and the shape of the underlying process distribution. A detailed discussion is given below.

If we consider the $M R L_{\delta}$ values for various symmetric distributions under different choices of $c_{l}$, for the $\operatorname{EX}(25)$ chart we usually see that the $M R L_{\delta}$ values initially decline as $c_{l}$ increases and reaches a minimum around $c_{l}=1$ for small to moderate shifts $(\delta=0.25$ and 0.50$)$ (see Figures 1a,b) and around $c_{l}=0.5$ for moderate to large shifts $(\delta=0.75$ and 1.00) (see Figures $1 \mathrm{c}, \mathrm{d}$ ). After the minimum is reached the $M R L_{\delta}$ values begin to increase sharply. Thus, for symmetric distributions, the NPCUSUM charts have a U-shape function when the $M R L_{\delta}$ is plotted against the reference value $k$ when the $25^{\text {th }}$ percentile of the reference sample is used. However, for the SymmMixN distribution, the U-shape only holds for small to moderate shifts $(\delta=0.25$ and 0.50$)$ (see Figures 1a,b). That being said, the minimum $M R L_{\delta}$ values, for the SymmMixN distribution, is at the same $c_{l}$ values as the other distributions for all shifts under consideration.

Subsequently, the graphs for the EX(40), EX(50), EX(60) and EX(75) charts are shown in Figures 2-5 for the symmetric distributions.

From Figure 2 we see that a U-shape is observed in all cases with the minimum at $c_{l}=1$, except for the non-normal distributions when the desired magnitude of shift to be detected is small $(\delta=0.25)$ (see Figure 2a). From Figure 3 it can been see that a U-shape isn't as prominent as with the NPCUSUM-EX chart based on smaller percentiles, but it is still seen in all cases for the normal distribution and it's very prominent for moderate to large shifts $(\delta=0.75$ and 1.00$)$ for the other distributions (see Figures 3c,d). For the EX(60) chart the U-shape isn't as prominent as with the NPCUSUM-EX chart based on smaller percentiles, as it is only clearly seen for the normal distribution when $\delta=0.50$ (see Figure $4 \mathrm{~b}$ ). In all other cases it seems that larger values of $k$ (where $c_{l} \geq 1$ and recall that $\left.k=c_{l} \delta S T D E V\left(U_{j, r}\right)\right)$ are to be preferred regardless of the size of this shift. Finally, for the EX(75) chart the U-shape isn't as prominent as with the NPCUSUM-EX chart based on smaller percentiles, as it is only clearly seen for the normal distribution when the shift is moderate to large ( $\delta=0.75$ and 1.00) (see Figures 5c,d). In all other cases it seems that larger values of $k$ are preferred regardless of the size of this shift.

Next we consider the asymmetric distributions (see Figures 6, 7 and 8). It should be noted that the OOC performance of gamma and lognormal distributions are very similarly and, consequently, the plots of $M R L_{\delta}$ values for of these two distributions almost overlap on each other in Figures 6 to 8 . We first consider the graphs for the $\operatorname{EX}(25)$ chart.

If we consider the $M R L_{\delta}$ values for various asymmetric distributions under different choices of $c_{l}$, for the $\mathrm{EX}(25)$ chart we usually see the U-shape pattern for $c_{l}$ vs $M R L_{\delta}$ plot for moderate to 
Figure 7. Out-of-control $M R L_{\delta}$ values for different asymmetric distributions under different choices of $c_{l}$ for the NPCUSUM-EX chart based on the $40^{\text {th }}$ percentile of the reference sample
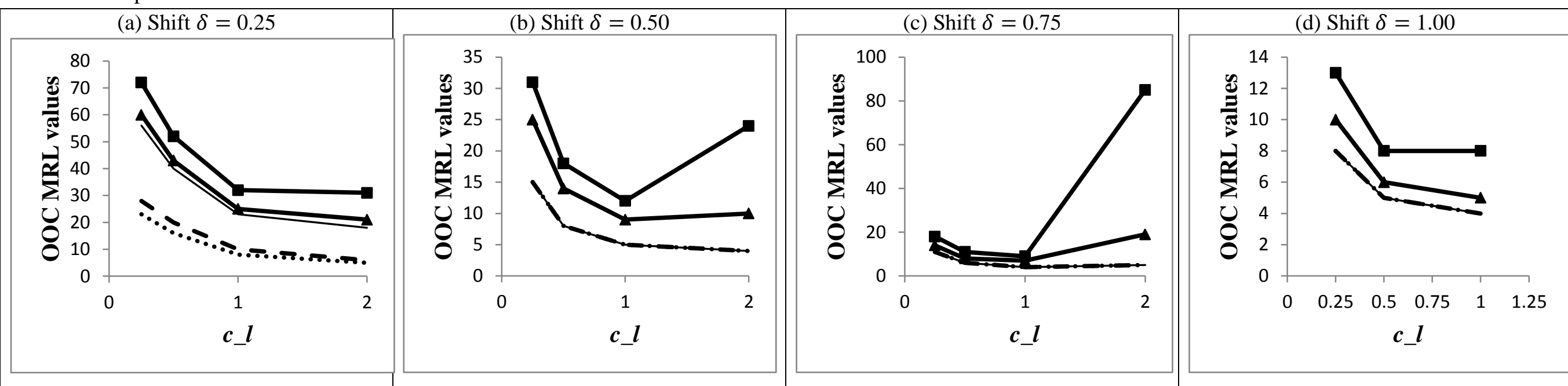

Note on Figure 7d. There is no marker for $c_{l}=2$, since the run-length characteristics could not be computed within a reasonable time, indicating that the run-length characteristics are extremely large and, consequently, $c_{l}=2$ would not be chosen.

Legend. $-\operatorname{EXP}(1) \cdots \cdots \operatorname{GAM}(0.5,1)-\operatorname{LogN} \rightarrow$ AsymmMixN1 $\rightarrow$ AsymmMixN2

Figure 8. Out-of-control $M R L_{\delta}$ values for different asymmetric distributions under different choices of $c_{l}$ for the NPCUSUM-EX chart based median of the reference sample

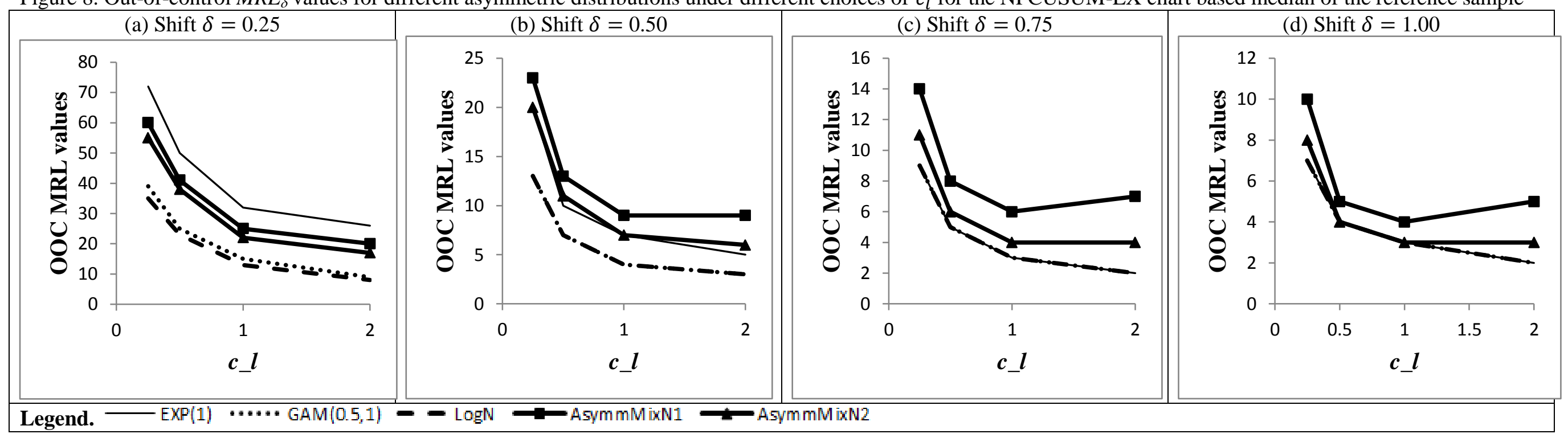


large shifts $\left(\delta=0.75\right.$ and 1.00) and $M R L_{\delta}$ reaches a minimum around $c_{l}=0.5$ (see Figures $6 \mathrm{c}, \mathrm{d}$ ). However, for a small shift ( $\delta=0.25$ ) the U-shape isn't as prominent for the exponential, gamma and lognormal distributions, having very long right tails and, consequently, larger values of $c_{l}$ are preferred. For a moderate shift of $\delta=0.50$ the minimum seems to be at $c_{l}=1.00$.

Similarly, for the EX(40) chart all the distributions have very long right tails and, consequently, larger values of $c_{l}$ are preferred when a small shift $(\delta=0.25)$ is in question. For moderate $(\delta=0.50$ and 0.75$)$ all distributions have the U-shape, except for the exponential, gamma and lognormal, again showing very long right tails and, consequently, larger values of $c_{l}$ are preferred $^{\star}$. For a large shift $(\delta=1.00)$ the U-shape is prominent for all distributions and it reaches a minimum at $c_{l}=1.00$ which, of course, is then the desired value to use.

From Figure 8 it is clear that for the EX(50) chart the U-shape isn't as prominent as with the NPCUSUM-EX based on smaller percentiles. All the distributions are showing very long right tails and, consequently, larger values of $c_{l}$ are preferred. This also holds for the $\operatorname{EX}(60)$ and $\operatorname{EX}(75)$ charts and, consequently, these figures are omitted to conserve space.

For the NPCUSUM-Rank chart we illustrate the shape of the $M R L_{\delta}$ profile graphically for the symmetric (see Figure 9) and asymmetric (see Figure 10) distributions. For the NPCUSUM-Rank chart the U-shape pattern for $c_{l}$ vs $M R L_{\delta}$ plot isn't as prominent as with the NPCUSUM-EX chart based on smaller percentiles, as it is only clearly seen for the normal distribution when the shift is moderate to large ( $\delta=0.75$ and 1.00) (see Figures 9c,d). In all other cases it seems that larger values of $k$ are to be preferred regardless of the size of this shift.

Overall, it is seen that the $\operatorname{EX}(25)$ chart performs exceptionally well in detecting smaller shifts when the underlying process distribution is exponential. The $\operatorname{EX(40)~chart~is~appropriate~for~}$ detection of smaller shifts when the underlying process distribution is lognormal or gamma and moderate shifts when the underlying process distribution is exponential. The $\operatorname{EX}(75)$ chart is the best for detecting larger shifts in almost all major univariate uni-modal bell-shaped distributions. Figures 1 to 8 and Tables 1 to 5 should be helpful for the practitioner in implementing the NPCUSUM-EX charts. To further assist the practitioner in implementing the chart and, more specifically, which value of $c_{l}$ to choose, a summary of the discussions above are given in Table 6 . The choice of $k$ values was also investigated for the NPCUSUM-Rank chart and it seems that, in most cases, larger values of $k$ are preferred regardless of the size of this shift for all distributions that are non-normal.

\footnotetext{
${ }^{\ddagger}$ Recall that $k=c_{l} \delta \operatorname{STDEV}\left(U_{j, r}\right)$.
} 
Figure 9. Out-of-control $M R L_{\delta}$ values for different symmetric distributions and under different choices of $c_{l}$ for the NPCUSUM-Rank chart

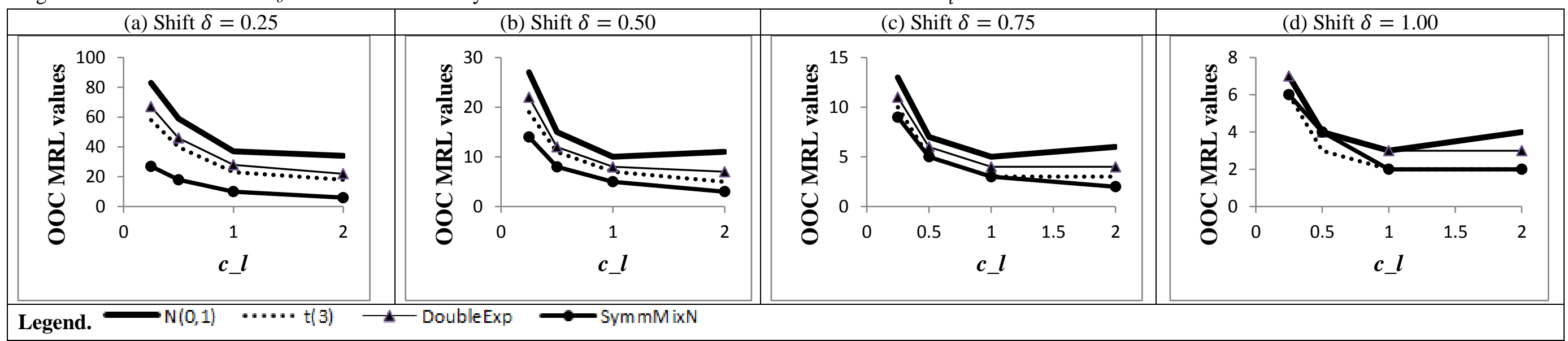

Figure 10. Out-of-control $M R L_{\delta}$ values for different asymmetric distributions and under different choices of $c_{l}$ for the NPCUSUM-Rank chart

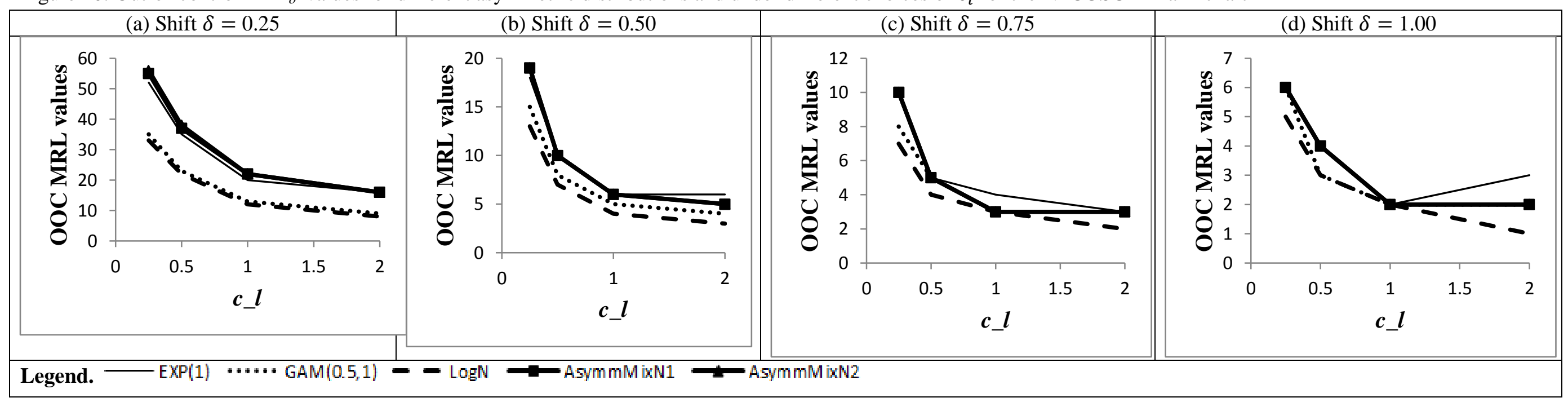


Table 6. Summary of the different reference sample percentiles and the choices of $c_{l}$

\begin{tabular}{|c|c|c|c|c|c|}
\hline \multicolumn{6}{|c|}{ If the practitioner has reason to suspect that the underlying process distribution is symmetric } \\
\hline Desired shift / Chart & EX $(25)$ & $\mathbf{E X}(40)$ & $\mathbf{E X}(50)$ & $\mathbf{E X}(60)$ & EX(75) \\
\hline 0.25 & \multirow{2}{*}{$c_{l}=1.00$} & \multirow{2}{*}{$c_{l}=1.00$} & \multirow{2}{*}{$\begin{array}{l}\text { Larger values of } \\
c_{l} \text { are preferred, } \\
\text { preferably } c_{l} \geq 1\end{array}$} & \multirow{4}{*}{$\begin{array}{l}\text { Larger values of } \\
c_{l} \text { are preferred } \\
\text { regardless of the } \\
\text { size of this shift, } \\
\text { preferably } c_{l} \geq 1\end{array}$} & \multirow{4}{*}{$\begin{array}{l}\text { Larger values of } \\
c_{l} \text { are preferred } \\
\text { regardless of the } \\
\text { size of this shift, } \\
\text { preferably } c_{l} \geq 1\end{array}$} \\
\hline 0.50 & & & & & \\
\hline 0.75 & \multirow[t]{2}{*}{$c_{l}=0.50$} & $c_{l}=1.00$ & \multirow[t]{2}{*}{$c_{l}=1.00$} & & \\
\hline 1.00 & & $c_{l}=0.50$ or 1.00 & & & \\
\hline \multicolumn{6}{|c|}{ If the practitioner has reason to suspect that the underlying process distribution is asymmetric } \\
\hline 0.25 & $\begin{array}{l}\text { Larger values of } \\
c_{l} \text { are preferred, } \\
\text { preferably } c_{l} \geq 1\end{array}$ & \multirow{2}{*}{$\begin{array}{l}\text { Larger values of } \\
c_{l} \text { are preferred, } \\
\text { preferably } c_{l} \geq 1\end{array}$} & \multirow{4}{*}{$\begin{array}{l}\text { Larger values of } \\
c_{l} \text { are preferred } \\
\text { regardless of the } \\
\text { size of this shift, } \\
\text { preferably } c_{l} \geq 1\end{array}$} & \multirow{4}{*}{$\begin{array}{l}\text { Larger values of } \\
c_{l} \text { are preferred } \\
\text { regardless of the } \\
\text { size of this shift, } \\
\text { preferably } c_{l} \geq 1\end{array}$} & \multirow{4}{*}{$\begin{array}{l}\text { Larger values of } \\
c_{l} \text { are preferred } \\
\text { regardless of the } \\
\text { size of this shift, } \\
\text { preferably } c_{l} \geq 1\end{array}$} \\
\hline 0.50 & $c_{l}=1.00$ & & & & \\
\hline 0.75 & \multirow{2}{*}{$c_{l}=0.50$} & \multirow{2}{*}{$c_{l}=1.00$} & & & \\
\hline 1.00 & & & & & \\
\hline
\end{tabular}




\section{Example}

We illustrate our proposals first using a well-known dataset from Montgomery (2001; Tables 5.1 and 5.2) on the inside diameters of piston rings manufactured by a forging process. The data given in Table 5.1 contains twenty-five retrospective or Phase I samples, each of size five, that were collected when the process was thought to be IC, i.e. $m=125$. An analysis in Montgomery (2001) showed that these data are from an IC process and thus can be considered to be Phase I reference data. Note also that for these data, a goodness of fit test for normality is not rejected. This does not guarantee that the normality assumption for a parametric CUSUM chart is valid but often the practical implication is as such. For the $\operatorname{EX}(25), \operatorname{EX}(40), \operatorname{EX}(50), \operatorname{EX}(60)$ and $\operatorname{EX}(75)$ charts we use $c_{l}=1.00$ so that $k=\delta S T D E V\left(U_{j, r}\right)$. For comparison purposes we include the NPCUSUM-Rank chart with $c_{l}=1.00$ so that $k=\delta \operatorname{STDEV}\left(W_{j}\right)$. For all the charts we use a search algorithm to find the value of $H$ such that $M R L_{0} \approx 350$. Note that, the values of the respective reference sample percentiles are as follows: $25^{\text {th }}$ percentile $=73.995,40^{\text {th }}$ percentile $=73.998$, median $=74.001,60^{\text {th }}$ percentile $=74.004$ and $75^{\text {th }}$ percentile $=74.008$. All of the measurements are in $\mathrm{mm}$.

In order to calculate the Phase II exceedance control charts, we use the data in Table 5.2 of Montgomery (2001) that contains fifteen prospective (Phase II) samples each of five observations $(n$ $=5$ ). The desired shift to be detected was taken to be small $\delta=0.25$ (see Figures 11 and 14 for the exceedance and rank charts, respectively), moderate $\delta=0.50$ (see Figures 12 and 15 for the exceedance and rank charts, respectively) and large $\delta=1.00$ (see Figures 13 and 16 for the exceedance and rank charts, respectively). Note that for Figures 11 to 13 the $\operatorname{EX(25)}$ and $\operatorname{EX(40)}$ charts weren't drawn, since they performed the worst overall. This also simplified the presentation in the figures (instead of 10 curves each figure now only has 8 curves). Also note that Figures 11 to 13 only show the exceedance charts and that the NPCUSUM-Rank charts are shown in Figures 14 to 16. The reason is that the vertical axes of the NPCUSUM-EX chart and that of the NPCUSUM-Rank chart differ dramatically and, accordingly, cannot be drawn on the same figure.

When the underlying process distribution is normal and we have a small shift $(\delta=0.25)$, Table 1 suggests that the NPCUSUM-Rank chart performs best followed by the EX(75) chart. From Figures 11 and 14 we can see that the EX(50) chart performs the worst and doesn't signal at all. The EX(60) chart almost signals, whereas the EX(75) chart actually does signal at sample number 14 with a counter of $N^{+}=6$, meaning that the shift most likely occurred at sample number 8 . The same is true for the NPCUSUM-Rank chart, i.e. it signals at sample number 14 with a counter of $N^{+}=6$, meaning that the shift most likely occurred at sample number 8. In conclusion, both the $\operatorname{EX}(75)$ chart and the NPCUSUM-Rank chart perform similarly and are the best. 


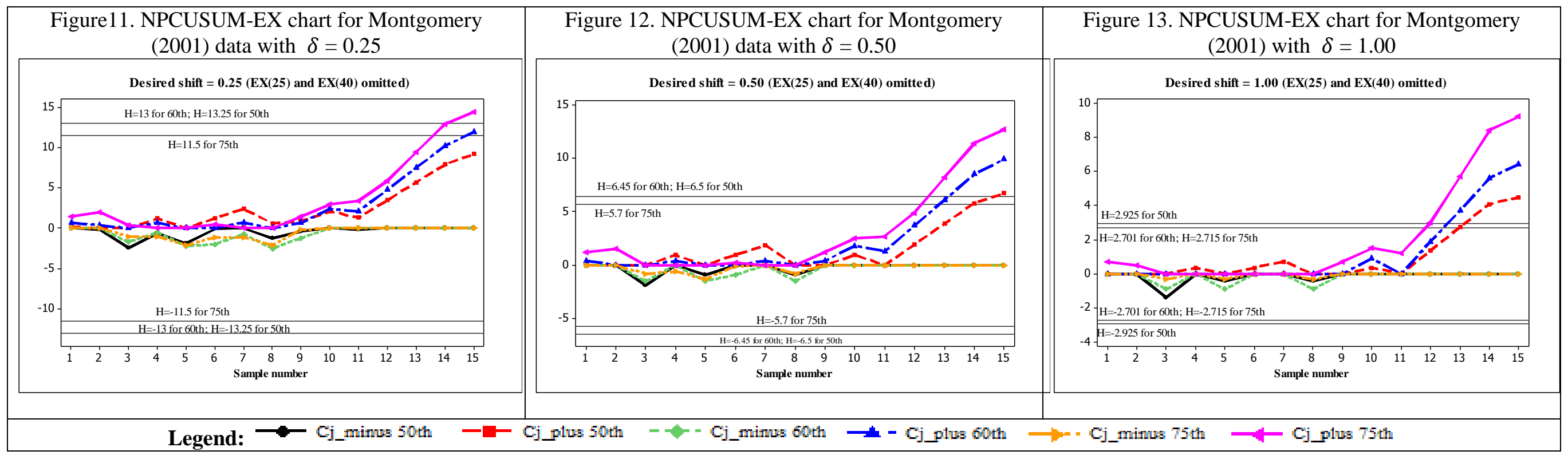

Figure 14. NPCUSUM-Rank chart for Montgomery (2001) data with $\delta=0.25$

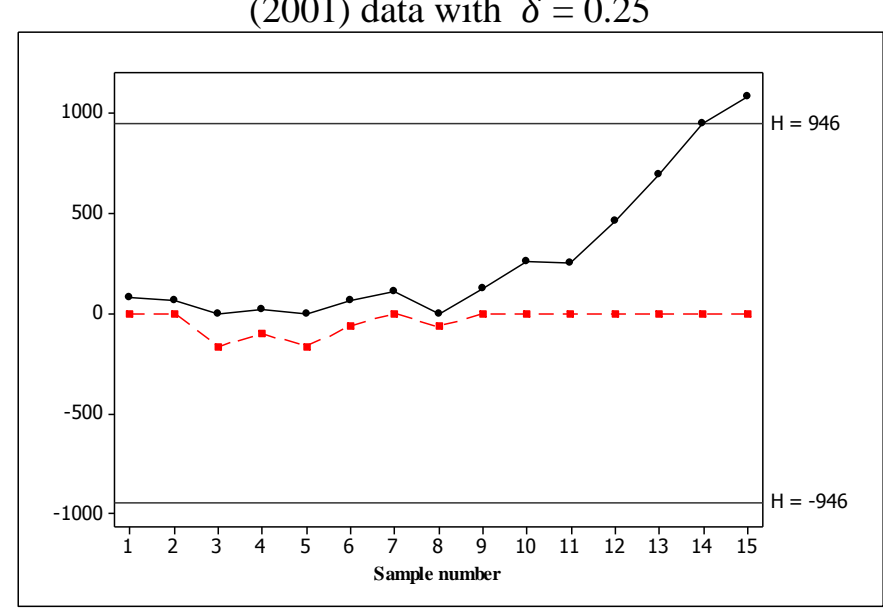

Figure 15. NPCUSUM-Rank chart for Montgomery (2001) data with $\delta=0.50$

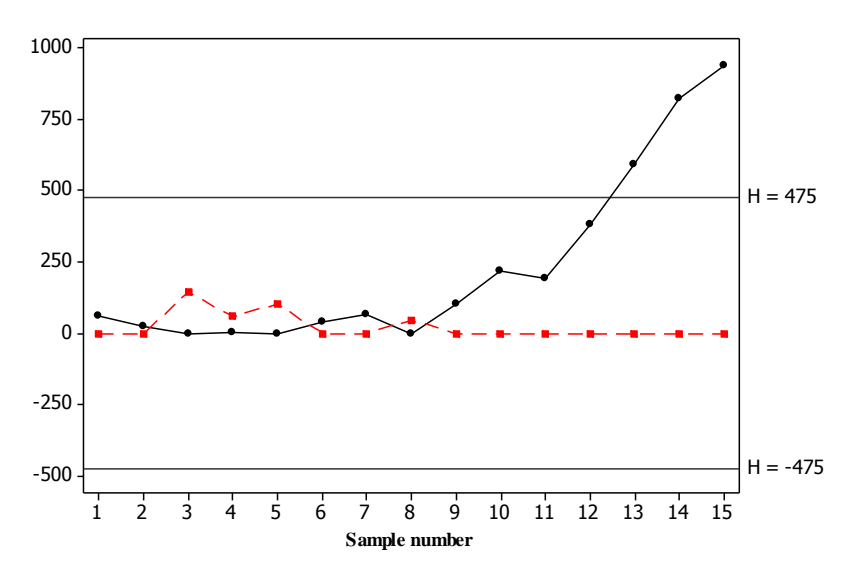

Legend: Solid line $=C_{j}^{+}$and Dotted line $=C_{j}^{-}$
Figure 16. NPCUSUM-Rank chart for Montgomery (2001) with $\delta=1.00$

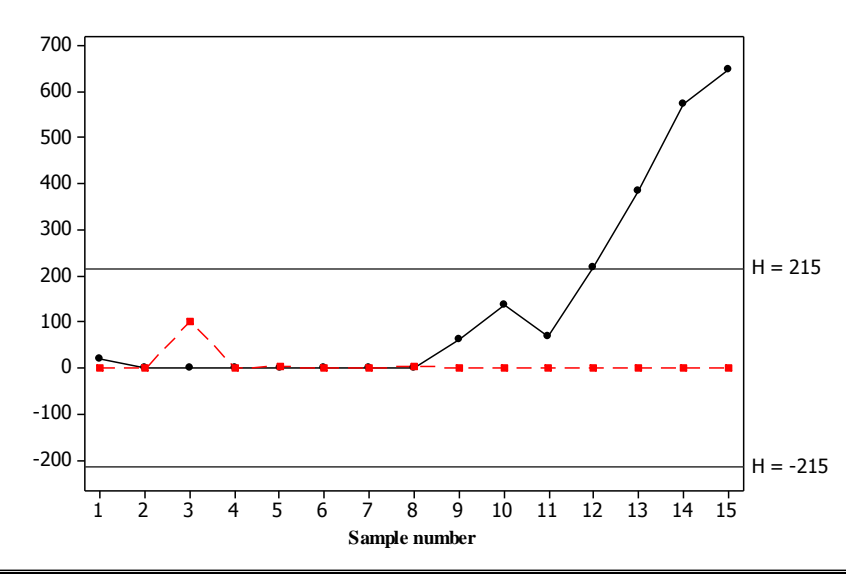


Again, for a normally distributed process and for a moderate shift $(\delta=0.50)$, Table 5 suggests that NPCUSUM-Rank performs best. From Figures 12 and 15 we can see that that the $\operatorname{EX}(50)$ chart signals at sample number 15 with a counter of $N^{+}=4$, indicating that the shift most likely occurred at sample number 11. The EX(60) chart signals at sample number 14 with a counter of $N^{+}=5$, indicating that the shift most likely occurred at sample number 9 . The EX(75) chart and the NPCUSUM-Rank chart signal at the earliest, at sample number 13, both with a counter of $N^{+}=$ 5 , indicating that the shift most likely occurred at sample number 8 . Thus, we find that the $\operatorname{EX}(75)$ chart and the NPCUSUM-Rank chart performs similarly and outperforms their competitors when tuned for a moderate shift $(\delta=0.50)$.

Further, if the process distribution is normal and we have a large shift $(\delta=1.00)$, Table 5 suggests that the NPCUSUM-Rank chart and the EX(75) chart perform similarly and the best. From Figures 13 and 16 we can see that the EX(50) chart signals on sample number 14 with a counter of $N^{+}=3$, indicating that the shift most likely occurred at sample number 11 . The $\operatorname{EX}(60)$ chart signals a little quicker, at sample number 13 with a counter of $N^{+}=2$, also indicating that the shift most likely occurred at sample number 11. The $\operatorname{EX(75)~chart~signals~at~sample~number~} 12$ with a counter of $N^{+}=3$, indicating that the shift most likely occurred at sample number 9. The NPCUSUM-Rank chart also signals at sample number 12 , however the counter is $N^{+}=4$, indicating that the shift most likely occurred at sample number 8 .

The findings above are very interesting, because the small shift $(\delta=0.25)$ goes nearly undetected, except by the NPCUSUM-EX chart based on the $75^{\text {th }}$ percentile and the NPCUSUMRank chart which signal, but only at the second last sample. Also, for the same data, the parametric CUSUM- $\bar{X}$ chart, with design parameters equal to $h=4$ and $k=0.5$ (a popular choice for the design parameters in the parametric setting providing a good IC performance) signals on sample number 12 (the reader is referred to the overview paper by Chakraborti et al. (2011) for details). This is the earliest signal, except for the NPCUSUM-Rank chart and the EX(75) chart designed for a large shift $(\delta=1.00)$ which also signal at sample number 12 . Thus, overall, the parametric CUSUM- $\bar{X}$ chart is performing best, which isn't surprising, as it is expected that parametric methods will outperform their parametric counterparts when all assumptions are met.

\section{Summary and Concluding Remarks}

The NPCUSUM charts offer an attractive alternative in practice as they combine the inherent advantages of nonparametric charts with the better small shift detection capability of CUSUM-type charts. We examine a class of NPCUSUM charts based on the exceedance statistic by investigating 

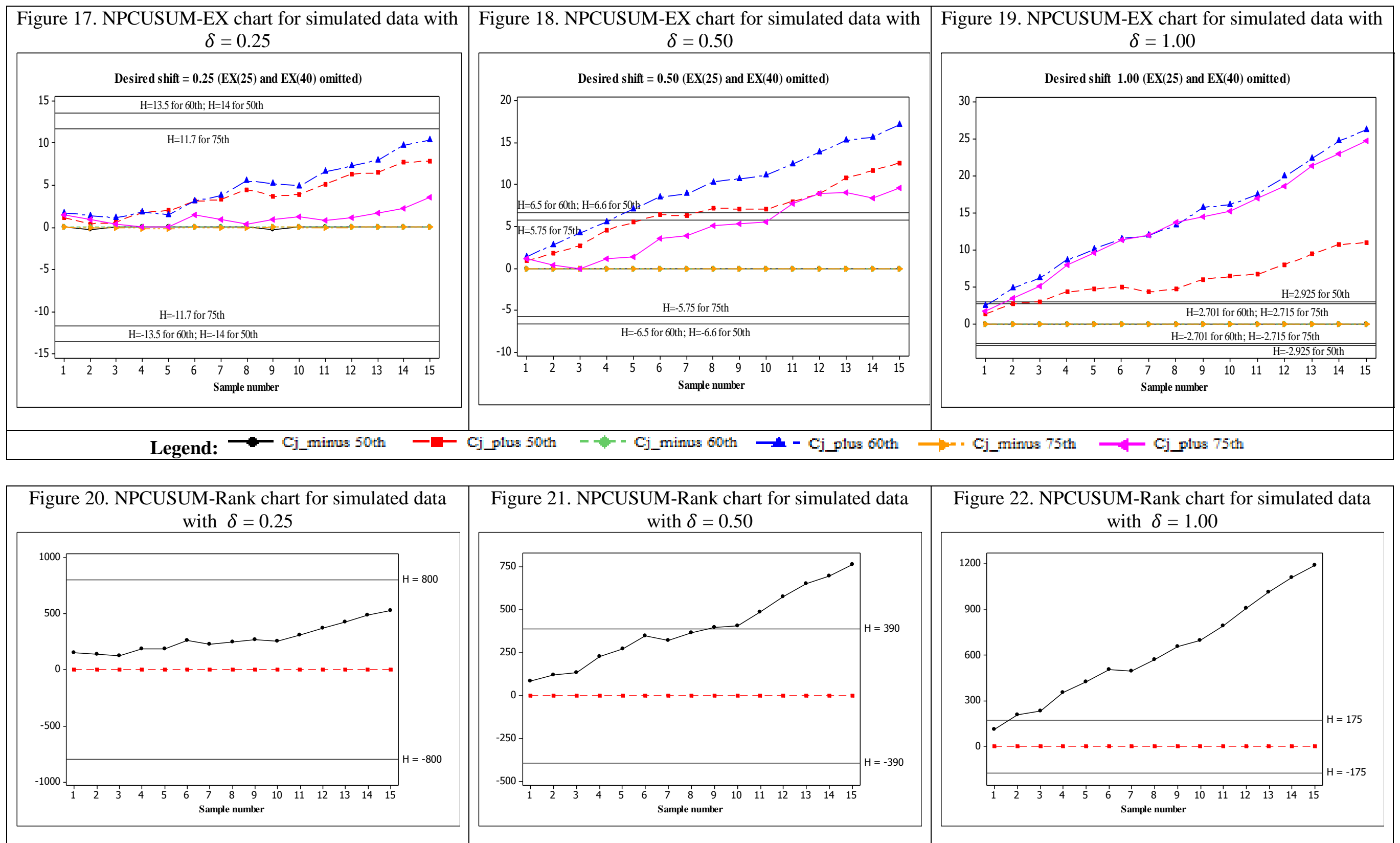
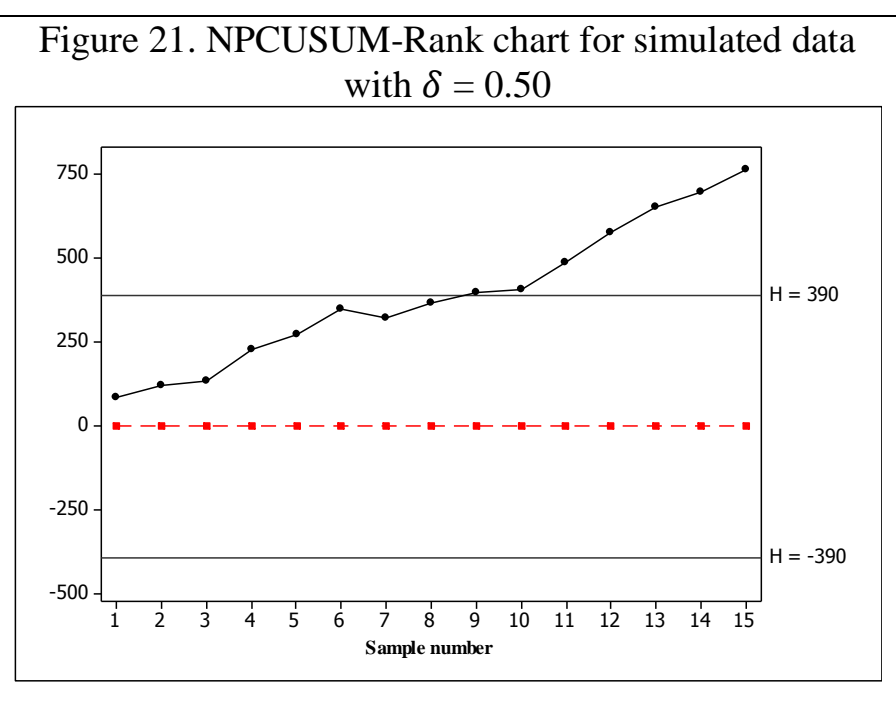

Legend: Solid line $=C_{j}^{+}$and Dotted line $=C_{j}^{-}$
Figure 22. NPCUSUM-Rank chart for simulated data with $\delta=1.00$

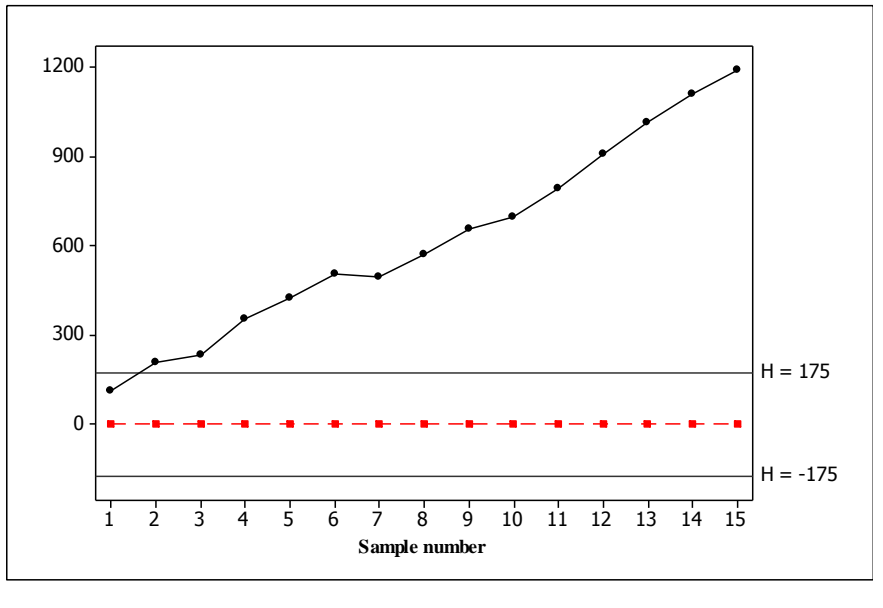


which order statistic (percentile), from the reference sample, should be used for good overall performance. The chart based on exceedance statistics is compared with the Wilcoxon rank-sum statistic based nonparametric chart. We see that in general, the exceedance CUSUM chart based on higher percentiles performs better in many cases for a number of common distributions. We also examine the impact of the reference value, $k$, on the performance of the chart and conclude that the NPCUSUM-EX chart, using the $75^{\text {th }}$ percentile of the reference sample, is a good overall chart for detecting a larger location shift. Other reference sample percentiles, such as the $25^{\text {th }}$ or the $40^{\text {th }}$, can also be used when a smaller shift in location is expected. More specifically, for moderate to large shifts there is little doubt that the practitioner should use the exceedance chart based on the $75^{\text {th }}$ percentile which signals quickly for all reference values under consideration. This is an interesting result in the literature on nonparametric exceedance/precedence tests and control charts. Note that our metric of comparison is the $M R L$, which we recommend over the $A R L$. In terms of future research, it would be interesting to compare the NPCUSUM-EX chart with some self-starting charts. The authors also wish to extend this work to the nonparametric exponentially weighted moving average control chart based on the exceedance statistic. This is currently being investigated and will be presented in a separate paper. Other possibilities for future research include studying the economic design of the NPCUSUM-EX chart and investing implementation procedure that will provide guarantee over unconditional Phase II run-length.

\section{References}

Barnard, G.A. 1959. Control charts and stochastic processes. Journal of the Royal Statistical Society, $B, 21$ (2), 239-271.

Chakraborti, S. and Graham, M.A. 2007. Nonparametric control charts. Encyclopedia of Statistics in Quality and Reliability, 1, 415 - 429, John Wiley, New York.

Chakraborti, S., Human, S.W. and Graham, M.A. 2011. Nonparametric (distribution-free) quality control charts. In Handbook of Methods and Applications of Statistics: Engineering, Quality Control, and Physical Sciences. N. Balakrishnan, Ed., 298-329, John Wiley \& Sons, New York.

Chakraborti, S., Van der Laan, P. and Van de Wiel, M.A. 2004. A class of distribution-free control charts. Journal of the Royal Statistical Society. Series C: Applied Statistics, 53 (3), 443-462.

Chatterjee, S. and Qiu, P. 2009. Distribution-free cumulative sum control charts using bootstrapbased control limits. The Annals of Applied Statistics, 3 (1), 349 - 369.

Goel, A.L. 2011. Cumulative sum control charts. In Handbook of Methods and Applications of Statistics: Engineering, Quality Control, and Physical Sciences. N. Balakrishnan, Ed., 120-129, John Wiley \& Sons, New York. 
Graham, M.A., Mukherjee, A. and Chakraborti, S. 2012. Distribution-free exponentially weighted moving average control charts for monitoring unknown location. Computational Statistics and Data Analysis, 56 (8), 2539 - 2561.

Hawkins, D.M. and Olwell, D.H. 1998. Cumulative sum charts and charting for quality improvement. Springer-Verlag, New York.

Jones, L. A., Champ, C. W. and Rigdon, S.E. 2004. The run length distribution of the CUSUM with estimated parameters. Journal of Quality Technology, 36 (1), 95-108.

Khoo, M.B.C. and Teh, S.Y. 2009. A study on the effects of trends due to inertia on EWMA and CUSUM charts. Journal of Quality Measurement and Analysis, 5 (2), 73-80.

Khoo, M.B.C., Wong, V.H., Wu, Z. and Castagliola, P. 2011. Optimal designs of the multivariate synthetic chart for monitoring the process mean vector based on median run length. Quality and Reliability Engineering International, 27 (8), 981-997.

Kim, S.H., Alexopoulos, C., Tsui, K.L. and Wilson, J.R. 2007. A distribution-free tabular CUSUM chart for autocorrelated data. IIE Transactions, 39 (3), 317-330.

Li, S.Y., Tang, L.C. and Ng, S.H. 2010. Nonparametric CUSUM and EWMA control charts for detecting mean shifts. Journal of Quality Technology, 42 (2), 209-226.

Liu, L., Tsung, F. and Zhang, J. 2013. Adaptive nonparametric CUSUM scheme for detecting unknown shifts in location. International Journal of Production Research. DOI: $10.1080 / 00207543.2013 .812260$

McDonald, D. 1990. A CUSUM procedure based on sequential ranks. Naval Research Logistics, 37 (5), 627-646.

Montgomery, D.C. 2001. Introduction to Statistical Quality Control, $4^{\text {th }}$ Edition, John Wiley, New York, NY.

Mukherjee, A., Graham, M.A. and Chakraborti, S. 2013. Distribution-free exceedance CUSUM control charts for location. Communications in Statistics - Simulation and Computation, 42 (5), 1153-1187.

Yang, S. and Cheng, S.W. 2011. A new non-parametric CUSUM mean chart. Quality and Reliability Engineering International, 27, 867-875. 